\title{
Bow Ties in the Sky. II. Searching for Gamma-Ray Halos in the Fermi Sky Using Anisotropy
}

\author{
Paul Tiede ${ }^{1}$, Avery E. Broderick ${ }^{1,2}$ (1) , Mohamad Shalaby ${ }^{1,2,3}$ (i) , Christoph Pfrommer $^{4,5}$ (1) , Ewald Puchwein ${ }^{6}$, Philip Chang ${ }^{7}$ (D), and \\ Astrid Lamberts ${ }^{8}$ (iD) \\ ${ }^{1}$ Department of Physics and Astronomy, University of Waterloo, 200 University Avenue West, Waterloo, ON, N2L 3G1, Canada \\ ${ }^{2}$ Perimeter Institute for Theoretical Physics, 31 Caroline Street North, Waterloo, ON, N2L 2Y5, Canada \\ ${ }^{3}$ Department of Physics, Faculty of Science, Cairo University, Giza 12613, Egypt \\ ${ }^{4}$ Leibniz-Institut für Astrophysik Potsdam (AIP), An der Sternwarte 16, D-14482 Potsdam, Germany \\ ${ }^{5}$ Heidelberg Institute for Theoretical Studies, Schloss-Wolfsbrunnenweg 35, D-69118 Heidelberg, Germany \\ ${ }^{6}$ Institute of Astronomy and Kavli Institute for Cosmology, University of Cambridge, Madingley Road, Cambridge, CB3 0HA, UK \\ ${ }^{7}$ Department of Physics, University of Wisconsin-Milwaukee, 3135 North Maryland Avenue, Milwaukee, WI 53211, USA \\ ${ }^{8}$ TAPIR, Mailcode 350-17, California Institute of Technology, Pasadena, CA 91125, USA \\ Received 2016 September 15; revised 2017 September 28; accepted 2017 October 8; published 2017 November 28
}

\begin{abstract}
Many-degree-scale gamma-ray halos are expected to surround extragalactic high-energy gamma-ray sources. These arise from the inverse Compton emission of an intergalactic population of relativistic electron/positron pairs generated by the annihilation of $\gtrsim 100 \mathrm{GeV}$ gamma rays on the extragalactic background light. These are typically anisotropic due to the jetted structure from which they originate (in the case of radio galaxies) or are oriented perpendicular to a large-scale intergalactic magnetic field (for blazar geometries). Here, we propose a novel method for detecting these inverse Compton gamma-ray halos based on this anisotropic structure that is centered on the active galactic nucleus (AGN). By marginalizing over the radial distribution of halo photons, we demonstrate that the angular power spectrum shows a characteristic sawtooth pattern with a dominant dipolar power and elevated even multipoles. Specifically, we show that by stacking those angular power spectra instead of images, it is possible to robustly detect gamma-ray halos with existing Fermi Large Area Telescope (LAT) observations for a broad class of intergalactic magnetic fields. Importantly, by testing a large number of systematics, our suggested power spectrum statistics appears to be robust and unbiased with respect to systematic uncertainties within the LAT instrumental response and associated with contaminating astronomical sources.
\end{abstract}

Key words: BL Lacertae objects: general - gamma rays: diffuse background - gamma rays: general - infrared: diffuse background - plasmas - radiation mechanisms: non-thermal

\section{Introduction}

The extragalactic gamma-ray sky at $\mathrm{TeV}$ energies is dominated by blazars, a subclass of active galactic nuclei (AGNs) with powerful relativistic outflows directed at us. The relativistic jets are powered by (inefficient) accretion onto a central nucleus, presumably a supermassive black hole at rates at least two orders of magnitude below the Eddington rate. Jetdominated AGNs can be subdivided into blazars (with the line of sight intersecting the jet opening angle) and non-aligned non-thermal-dominated AGNs such as radio galaxies (Urry \& Padovani 1995). As a result, the gamma-ray emission of blazars benefits from relativistic Doppler boosting, shifting the upper end of the gamma-ray emission into the $\mathrm{GeV} / \mathrm{TeV}$ energy regime. Blazars exhibit a continuous sequence: their luminosity anti-correlates with the peak energy of their synchrotron spectrum, i.e., the objects emitting very high-energy gammarays (VHEGRs) at $\mathrm{TeV}$ energies have the lowest intrinsic luminosity (e.g., Fossati et al. 1998; Ghisellini et al. 1998).

AGNs exhibit a strong redshift evolution (as manifested in the evolution of quasars and radio galaxies) with a steeply rising comoving luminosity density up to a redshift $z \approx 2$ and a decline thereafter (Hopkins et al. 2007). In contrast, we can only observe nearby $\mathrm{TeV}$ blazars that typically reach redshifts of $z \lesssim 0.3$ (Wakely \& Horan 2008). ${ }^{9}$ The reason for this apparent contradiction lies in the low luminosity of $\mathrm{TeV}$ blazars and the

\footnotetext{
See http://tevcat.uchicago.edu, catalog version 3.4
}

finite mean free path of $\mathrm{TeV}$ photons as they propagate through space (Ackermann et al. 2012b; Domínguez et al. 2013), precluding the detection of high-redshift blazars (if they exist). The opacity of the universe to $\mathrm{TeV}$ photons is due to the annihilation and pair production of $\mathrm{TeV}$ photons of energy $E_{\gamma}$ on the extragalactic background light (EBL; Gould \& Schréder 1967; Salamon \& Stecker 1998), with a VHEGR mean free path of $D_{\mathrm{pp}}\left(E_{\gamma}, z\right) \approx 130 \ldots 6 E_{6 \mathrm{TeV}}^{-1} \mathrm{Mpc}$ for redshifts $z=$ 0 ... 1 (Neronov \& Semikoz 2009). Momentum conservation ensures that the pairs propagate essentially in the same direction as the parent $\mathrm{TeV}$ photon, and energy conservation implies a pair energy of $E_{e^{ \pm}} \approx E_{\gamma} / 2$.

The resulting ultrarelativistic pairs of electrons and positrons are commonly assumed to lose energy primarily through inverse Compton (IC) scattering with photons of the cosmic microwave background (CMB), cascading the original $\mathrm{TeV}$ emission down to (multi-) $\mathrm{GeV}$ energies on a mean free path for the scattering process of

$$
D_{\mathrm{IC}}=\frac{3 m_{e}^{2} c^{4}}{4 \sigma_{\mathrm{T}} u_{\mathrm{CMB}} E_{e^{ \pm}}} \approx 0.1\left(\frac{E_{e^{ \pm}}}{3 \mathrm{TeV}}\right)^{-1}(1+z)^{-4} \mathrm{Mpc}
$$

where $m_{e} c^{2}$ is the electron rest mass energy, $\sigma_{\mathrm{T}}$ is the Thompson cross section, and $u_{\mathrm{CMB}}$ is the CMB energy density.

However, the IC cascaded (ICC) multi-GeV emission has not been observed by the Fermi Large Area Telescope (LAT), indicating that some additional physics needs to be considered (see, e.g., Neronov \& Vovk 2010; Tavecchio et al. 2010, 2011; 
Dermer et al. 2011; Dolag et al. 2011; Taylor et al. 2011; Takahashi et al. 2012; H.E.S.S. Collaboration et al. 2014; Prokhorov \& Moraghan 2016). The presence of intergalactic magnetic fields (IGMFs) would deflect the beam of $e^{+} / e^{-}$pairs with a Larmor radius of

$$
r_{\mathrm{L}}=\frac{E_{e^{ \pm}}}{e B} \approx 30\left(\frac{E_{e^{ \pm}}}{3 \mathrm{TeV}}\right)\left(\frac{B}{10^{-16} \mathrm{G}}\right)^{-1} \mathrm{Mpc}
$$

out of our line of sight. This reduces the ICC flux and thus provides a lower limit on the strength $B$ of the IGMF. For the associated ICC photons with energies of

$$
E_{\mathrm{IC}} \approx 2 \gamma^{2} E_{\mathrm{CMB}} \approx 70\left(\frac{E_{e^{ \pm}}}{3 \mathrm{TeV}}\right)^{2} \frac{E_{\mathrm{CMB}}}{1 \mathrm{meV}} \mathrm{GeV},
$$

the Fermi angular resolution is $\theta \approx 0.2$ or $3 \times 10^{-3}$ rad (using the $1 \sigma$ containment angle of Combined events; see Figure 57 of Ackermann et al. 2012a). Hence, a deflection of the pairs by an angle $D_{\mathrm{IC}} / r_{\mathrm{L}}>\theta$ implies a lower limit on the IGMF of $B \gtrsim 10^{-16} \mathrm{G}$ (see, e.g., Neronov \& Vovk 2010), with important implications for primordial magnetogenesis (for reviews, see Kandus et al. 2011; Durrer \& Neronov 2013).

Alternatively, ultrarelativistic pairs propagating through the intergalactic medium is an example of a beam-plasma system and thus is subject to beam-plasma instabilities (Broderick et al. 2012; Schlickeiser et al. 2012; Chang et al. 2016), which grow faster than the pair cooling rate due to IC scattering in the linear regime. If this result applies to the regime of nonlinear saturation (Schlickeiser et al. 2013; Chang et al. 2014; Shalaby et al. 2017; but see also Miniati \& Elyiv 2013; Sironi \& Giannios 2014), this causes the kinetic energy of the pairs to be transferred to the unstable electromagnetic modes in the background plasma. As this energy is dissipated, it heats the intergalactic medium, with important consequences for cosmological structure formation (Chang et al. 2012; Pfrommer et al. 2012; Puchwein et al. 2012; Lamberts et al. 2015), blazar luminosity function, and extragalactic gamma-ray background (Broderick et al. 2014a, 2014b).

How can we determine the ultimate fate of these pairs? Clearly, an unambiguous detection of the deflected pair halo emission would immediately prove the deflection hypothesis. So far, all work has concentrated on measuring the excess halo power at large angular scales through stacking analyses of blazar images. However, those resulted in null results (e.g., Ackermann et al. 2013a) despite some earlier claims (Ando \& Kusenko 2010), which were subsequently disproven (Neronov et al. 2011) because of the uncertainty of the exact shape and side lobes of Fermi's point spread function (Ackermann et al. 2012a). A more recent attempt (Chen et al. 2015) utilizes the most recent PSF; nevertheless, it suffers from similar sensitivities to the uncertain instrument response.

Here, we report a novel method for extracting signatures of the ICC component that exploits the large degree of anticipated anisotropy (see, e.g., Neronov et al. 2010; Long \& Vachaspati 2015; Broderick et al. 2016). This is caused by either the structure of the initial VHEGR jet (if its opening angle is not intersecting the line of sight) or the fact that both electrons and positrons are deflected in opposite directions by an IGMF. The details of the structure depend on both the mechanism and properties of the IGMF. However, in both cases, they can produce halos that are dominated by bilobed features. Such

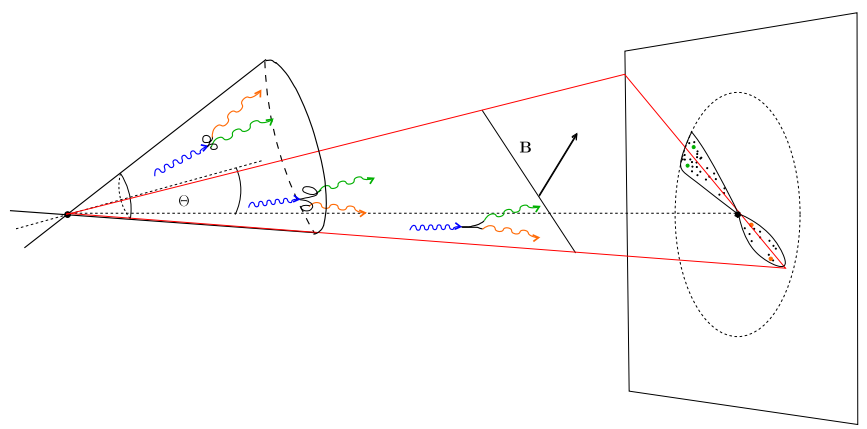

Figure 1. Cartoon of the mechanisms by which anisotropy in the ICC halos is generated for an IGMF that is uniform across the gamma-ray jet (IGMF coherence length $\lambda_{B} \gg 100 \mathrm{Mpc}$ ). The anisotropy is due to the geometry of the gyrating, relativistic pairs.

features are generic for the gamma-ray bright blazars observed by Fermi (Broderick et al. 2016).

In the standard picture of ICC halo formation, VHEGRs emitted from an AGN travel cosmological distances prior to generating energetic electron-positron pairs on the EBL, which then IC upscatter the $\mathrm{CMB}$ to $\mathrm{GeV}$ energies. However, for two independent reasons, these ICC halos are anisotropic. First, the VHEGRs are originally beamed along the jet axis. This is evidenced by the overwhelming dominance of blazars in the extragalactic gamma-ray AGN sample (Ackermann et al. 2011, 2015b). Because the VHEGR mean free path is long in comparison to the distance traveled during the IC cooling time of the resulting pairs, this implies that the emission is essentially local and therefore arises from a pair of conical regions indicated by the radio jet of the source AGN. If the IC gamma rays are isotropically emitted, arising, e.g., from a highly tangled IGMF, the spatial structure in gamma rays generates a corresponding structure in the $\mathrm{GeV}$ image.

Second, the process of gyration in the IGMF can also impart structure to the image. In the presence of an IGMF that is homogeneous on scales comparable to $D_{\mathrm{pp}}$, electrons and positrons will gyrate on fixed trajectories that emit toward an observer only for a subset of initial injection positions. This is still superimposed on the jet structure, resulting in a potentially asymmetric image structure, shown in Figure 1. Gamma rays on opposite sides of the original AGN are produced predominantly by different lepton species, i.e., positrons on one side and electrons on the other. ${ }^{10}$ It is this mechanism that is the subject of our efforts here.

In principle, this angular asymmetry can improve our ability to detect ICC halos in two different ways. First, the anisotropy implies a larger surface brightness for the ICC emission, and therefore, if we can properly orient the images, any excesses can be detected with a higher significance over the putative symmetric backgrounds. In practice, we do this not by rotating and stacking images, which proves to be infeasible due to the inability to determine the orientation of the deflecting magnetic field, but rather by constructing and stacking orientationindependent measures of the angular anisotropy in the gammaray sky about individual sources. In particular, we propose to exploit the pair halo anisotropy by computing suitably defined

\footnotetext{
${ }^{10}$ Strictly speaking, the identification of lobe sides with particular leptons does assume that the gyration timescale is long in comparison to the IC cooling timescale. Should this be the case today, it will continue to be true at higher redshift. Note that even if the IC cooling time is longer than the gyration period, the halo will still exhibit the bimodal structure.
} 
angular power spectra and stacking those. This approach is routinely applied in cosmological data analyses to determine cosmological parameters (e.g., from the CMB anisotropies)the key idea consists of averaging over the (unknown) orientation of the phases and accumulating signal in the multipoles of the dominant angular structures. ${ }^{11}$

Second, averaged over the long live time of the Fermi LAT, the resulting PSF is very nearly isotropic. A small residual angular structure in the PSF arising from the cubical geometry of the LAT enters at the hexadecapole $(m=4)$ order. As a result, unlike searches that focus on radial excesses, schemes that exploit the nearly quadrupolar nature of the halos are easily distinguished from systematic effects due to the PSF. Although this paper presents the detailed methodology and addresses the (known) systematics, we apply our blind experiment to Fermi LAT data in a companion paper (Tiede et al. 2017).

Of particular practical importance is the fact that the Fermi data set is not easily repeatable. As a result, we expended considerable effort to predict the anticipated anisotropy signal from various ICC halo models, and to design and optimize the construction of ICC halo diagnostics prior to analyzing the Fermi data. Here, we report the results of this optimization investigation, i.e., the manner in which mock realizations were produced (Section 2), a cursory description of the characteristics of the Fermi hard gamma-ray blazar sample (Section 3), the investigation of potential confounding features (Section 4), and the confidence levels with which the various ICC models could be excluded given a null result (Section 5). It is in this last step that the dividends of having done so become manifest-by training the analysis on simulated data (only weakly informed by the gross properties of the Fermi sample), we ensure that the resulting conclusions are governed by a priori statistics and are therefore well understood.

\section{Generating Mock Images of Gamma-Ray Sources}

Key to assessing any scheme to detect ICC halos is the creation of credible theoretical realizations of gamma-ray images of potential sources. Section 1 introduced the qualitative reasons to expect anisotropic structure in the ICC halo structure; how to do this quantitatively was presented in Broderick et al. (2016), which we summarize here.

The energies of the gamma rays that comprise the putative ICC halos typically lie below $100 \mathrm{GeV}$; much higher energy gamma rays are absorbed on the EBL. Below $1 \mathrm{GeV}$, the Fermi LAT PSF typically broadens substantially, limiting efforts to find asymmetric features and extending the contaminating influence of bright sources. Between $1 \mathrm{GeV}$ and $100 \mathrm{GeV}$, the Fermi LAT response functions only modestly depend on energy. Therefore, we restrict our attention to this energy range.

At the most granular level, Fermi images consist of collections of individual photons, numbering in the thousands for a single bright source, each with a reported sky location and energy. Thus, in principle, this procedure consists of first, identifying the joint probability distribution of photons from various emission components with a given energy and location,

\footnotetext{
11 A similar strategy is described in Duplessis \& Vachaspati (2017), where the utility of the $Q$ statistic, a quantity that is closely related to what we describe as the quadrupolar power, is explored. A key distinction between the approaches presented here and in Duplessis \& Vachaspati (2017) is that we also construct and utilize the power at the other multipoles, providing an independent characterization of potential systematic errors.
}

$d F / d E d^{2} x$, and second, efficiently drawing a random realization from this, $\left\{E_{j}, \boldsymbol{x}_{j}\right\}$. In practice, this is further modified by the Fermi LAT response, which primarily impacts the images via the PSF. We consider a three-component model comprised of a uniform background, an intrinsic point source, and a putative ICC halo. The former two are well-defined and have parameters fixed by Fermi directly. Less clear are the ICC halos. Their brightness and morphology depend on the poorly constrained VHEGRs, and thus require some spectral and collimation model that extends the known Fermi properties to $\mathrm{TeV}$ energies. This introduces a variety of additional poorly known parameters.

In addition to the intrinsic parameters of the source, the structure of the ICC halo depends critically upon the assumed geometry of the IGMF (Broderick et al. 2016). In principle, this corresponds to different assumptions about the IGMF power spectrum. In practice, the different limiting structures-small scale, tangled and large scale, uniform-imply distinct evolution models for the ultrarelativistic electron-positron pairs following their generation by VHEGR photons from the gamma-ray blazars; the pairs' momenta are rapidly isotropized in the former case, while pairs gyrate around the magnetic field in the latter case, emitting (toward the observer) only when their momentum is directed toward the observer.

Because near the jet axis, which is the line of sight for the gamma-ray bright blazar sources we consider here, there is little asymmetry in small, tangled IGMFs, we focus here on the other limit, i.e., the large-scale IGMF that is uniform across the extent of the gamma-ray jet. In principle, this requires uniformity on scales of $D_{\mathrm{pp}}$. In practice, this is reduced for nearby objects (e.g., Mkn 421) and at high energies; in combination, these typically require $\lambda_{B} \gg 100 \mathrm{Mpc}$, where $\lambda_{B}$ is the IGMF coherence length. Unlike the small-scale, tangled IGMF, there is no condition on the magnetic field strength a priori. However, weak fields necessarily produce more compact image features as a result of their smaller deflection angles. To produce an observable ICC halo feature, i.e., one that extends beyond 0.6 , requires

$$
B \gtrsim 4 \times 10^{-17}\left(\frac{E_{\mathrm{IC}}}{10 \mathrm{GeV}}\right)(1+z)^{4} \mathrm{G} .
$$

For smaller magnetic fields, the ICC halo structure will typically be overwhelmed by the direct emission component and its anisotropy substantially degraded by the Fermi PSF. These produce ICC halos that for the Fermi blazars are characterized by strong anisotropy with an extent dictated by the magnetic field strength, an example of which is shown in Figure 2. As described in detail within Broderick et al. (2016), this is due to the intrinsic anisotropy in the pair distribution function and the strong beaming of the IC emission.

We note that large-scale volume-filling fields are difficult to generate. Generally, a volume-filling IGMF is likely due to a primordial field that arises from electroweak or quantum chromodynamics phase transitions in the early universe or during cosmic inflation. Although the general nature of these fields in still unknown, causal constraints limit the coherent length scales of primordial fields generated from post-inflation mechanisms, e.g., phase transitions, to small comoving scales, typically <10 kpc (Widrow et al. 2012). These small-scale 

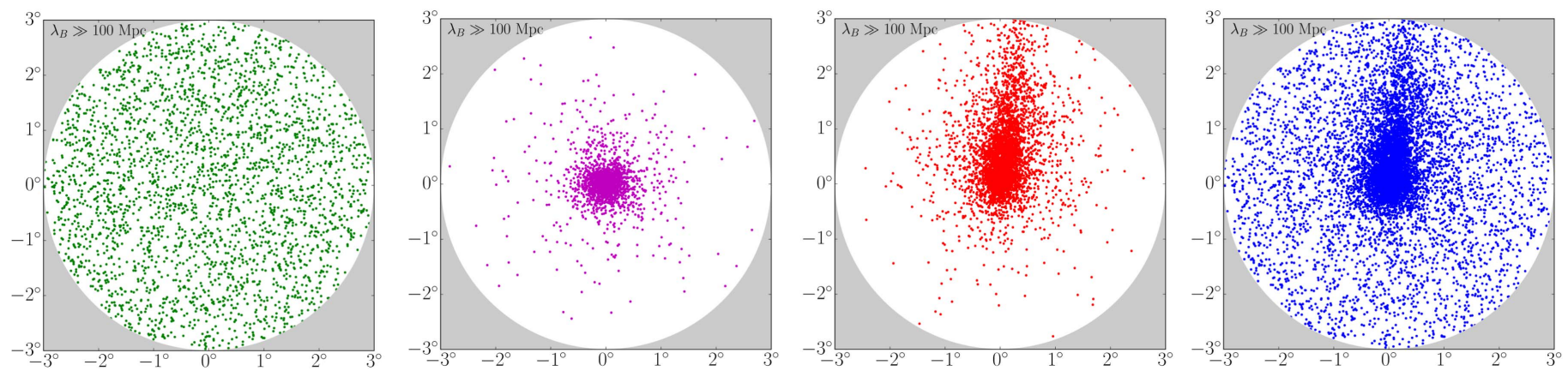

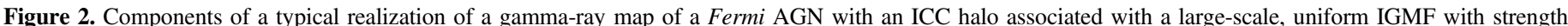

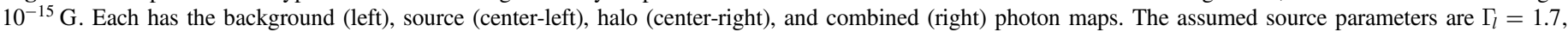

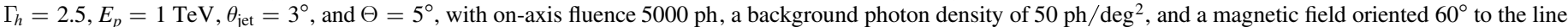
of sight and $170^{\circ}$ from the horizontal axis.

fields may inverse cascade to large-scale fields up to $\sim 0.1 \mathrm{Mpc}$, i.e., the sonic scale of $10^{4} \mathrm{~K}$ gas over the age of the universe (Ryu et al. 2012), but is notably smaller than the fields we constrain in this work.

To generate large-scale ( $\gtrsim 1 \mathrm{Mpc}$ ) volume-filling fields, seed fields must have been generated prior to or during inflation (Turner \& Widrow 1988) or from second-order interactions between the photon and electron fluid prior to recombination (Ichiki et al. 2006). In the former case, additional physics of super-adiabatic magnetic amplification (Tsagas \& Kandus 2005) are needed to keep these fields relevant today. This leaves the strength of these inflationary fields unconstrained (Widrow et al. 2012). In the latter case, the expected comoving field strength is $B \sim 10^{-24} \mathrm{G}$. Keeping in mind that the physics of the origin of the IGMF is completely unknown, we nevertheless focus on the large-scale case in this work for reasons of simplicity and constraining power.

We are ultimately interested in producing mock realizations of the Fermi sky, which we assume is comprised of a halo, direct emission, and background components. Thus, the mock Fermi images in the presence of a large-scale IGMF are characterized by 11 quantities (Broderick et al. 2016):

1. The source redshift, $z$;

2. The $1 \mathrm{GeV}-100 \mathrm{GeV}$ fluence, $N_{35}$;

3. The low-energy photon spectral index, $\Gamma_{l}$;

4. The high-energy photon spectral index, $\Gamma_{h}$;

5. The energy of the spectral break, $E_{p}$;

6. The gamma-ray jet opening angle, $\theta_{\text {jet }}$;

7. The gamma-ray jet viewing angle, $\Theta$;

8. The local background photon density, $N_{B}$;

9-11. The IGMF, $\boldsymbol{B}$.

As before, some of these are obtained from values reported for a subsample of Fermi AGNs while others must be sampled from the appropriate distributions; these are discussed in detail in Section 3. In addition we must define $\boldsymbol{B}$. While we will review this in Section 3.4, here we note that we do this by specifying independently an orientation and magnitude with the latter set via the current IGMF strength, $B_{0}$.

\subsection{Fermi Point Spread Function}

We assume the same PSF as described in Broderick et al. (2016), to which we direct the interested reader for details on implementation, and only summarize salient points here.
Because ICC halos have yet to be unambiguously detected, we consider the Pass 8R2_V6 ULTRACLEANVETO photon sample; these are the photons that are confidently associated with an astronomical origin and not necessarily nearby bright sources. The form of the Pass 8R2_V6 PSF is described in Broderick et al. (2016) and for the events of interest here is substantially simplified by the weak PSF dependence on energy above $1 \mathrm{GeV}$ and the fact that the collection of events within the Pass 8R2_V6 ULTRACLEANVETO sample is distributed among a large number of potential bore angles. As a result, the collective PSF for the Front and Back detectors are well approximated for each by the PSF at a single bore angle bin, corresponding to $36^{\circ} .9-45^{\circ} .6$ in both cases (for details see Broderick et al. 2016).

In principle, the square geometry of the LAT imposes a strong dependence on the azimuthal angle of the photon (Ackermann et al. 2012a). However, in practice, the long duration of the Fermi observations (eight years) combined with the solar tracking and eightfold symmetry results in a nearly cylindrical symmetry (Ackermann et al. 2012a). This may be broken for short duration or bursty events, and thus if the gamma-ray AGN of interest underwent periods of substantial variability, a small residual angular structure may appear. However, as discussed in Appendix D, such a structure will enter first at the hexadecapole, i.e., $m=4$, mode, and therefore is easily distinguishable from that due to the ICC halo structure.

\subsection{Source-Halo-Background Confusion}

The direct emission from the source, background, and ICC halo are not spatially distinct. For large-scale uniform IGMF geometries in particular, the halos are strongly centrally concentrated, and therefore will suffer from confusion with the source photons. Therefore, applying the observational constraints provided by the known source and background source counts requires a method to partition the ICC halo component between the source and background in a selfconsistent manner.

The LAT PSF provides a natural definition of those events that would be identified as "source" photons. Any substantial emission component beyond the $68 \%$ containment radius of the Pass 8R2_V6 ULTRACLEANVETO PSF would be identified as extended and therefore not included in the point-source flux estimates. This is also consistent with the energy- and detectordependent mask that we apply to the images to reduce Poisson noise (see Section 4.3). 
Therefore, we implicitly set the normalization of the ICC halo component by generating image realizations with an appropriate number of "source" photons inside the appropriate $68 \%$ containment radius, including those from all components. For strong sources with weak halos, this makes little difference. For weak sources with strong halos (e.g., those with very hard VHEGR SEDs), this curtails the halo emission appropriately.

Extending the "source" region farther begins to rapidly increase the angular size of the region as a result of the broad power-law tails on the Pass 8R2_V6 ULTRACLEANVETO PSFs. However, we verified that extending this to the $95 \%$ containment radius makes little difference to our ability to detect ICC halos.

\subsection{Near-source Halo Suppression}

A small subset of Fermi AGNs are closer than $D_{\mathrm{pp}}$, and therefore the assumption that the sources were sufficiently far for the full ICC halo is violated. It is possible to generate ICC halo models in this case for which the region contributing to the ICC halos is restricted to that between the Earth and the source. However, for the large-scale, uniform IGMF models, the smallangle contributions to the ICC halos are nearly uniformly distributed along the line of sight, enabling a simpler optical depth correction. That is, we reduce the anticipated halo flux by the energy-dependent factor $1-e^{-D_{P} / D_{\mathrm{pp}}}$, where $D_{P}$ is the proper distance to the VHEGR source.

\subsection{Time Delays and Duty Cycles}

Generally, the contributions to the ICC halos at different positions on the sky are not contemporaneous-there is a delay between ICC halo gamma rays produced along the line of sight and those off it. The typical delay times, $\delta t$, are geometric in nature and therefore correlated with the angular diameter distance $D_{A}$ from the central gamma rays source,

$$
\delta t \approx \frac{D_{A}^{2}}{2 D_{\mathrm{pp}} c} \theta^{2}
$$

where $\theta$ is the angular size of the observed halo. Therefore, the magnitude of the delay anticipated is limited by the size of the ICC halo. For ICC halos from gamma-ray blazars, the ICC halo is limited by both the size of the magnetic field deflections and the width of the gamma-ray jet. For the latter, this gives $\theta \lesssim \theta_{\text {jet }} D_{\mathrm{pp}} / D_{A}$, hence conservatively

$$
\delta t \lesssim \frac{D_{\mathrm{pp}}}{2 c} \theta_{\text {jet }}^{2}
$$

For a typical $\theta_{\text {jet }} \approx 3^{\circ}$ and $D_{\text {pp }} \approx 300 \mathrm{Mpc}$ at $z=0.3$ for a TeV VHEGR, this gives $\delta t \lesssim 10^{6}$ years. Although considerably larger than the present observing time, this is comfortably short in comparison to the typical radio duty cycles of a few times $10^{7}$ years to a few times $10^{8}$ years (Alexander \& Leahy 1987; McNamara et al. 2005; Nulsen et al. 2005; Shabala et al. 2008), suggesting that the current gamma-ray flux is indicative of that responsible for a putative ICC halo.

\section{The Fermi Gamma-Ray Blazar Targets}

Here, we summarize a variety of essential properties of the observed Fermi sample of bright, nearby gamma-ray blazars, i.e., objects likely to have detectable ICC halos. Among these are the intrinsic source parameters, e.g., observed flux, redshift, etc., as well as the extrinsic source context, e.g., local gammaray background, PSF, and the putative IGMF. Some of these can be specified for each source explicitly; others must be determined probabilistically. The list of targets that met all requirements within the Fermi Pass 8R2_V6 ULTRACLEANVETO class is presented in Table 1.

Because ICC halos are essentially reprocessed VHEGR emission, they exist solely around VHEGR-bright objects. Therefore, the goal of initial source class identification is to estimate the VHEGR brightness of individual gamma-ray AGNs. To do this, we exploit the 2FHL, which is optimized for the detection of objects above $50 \mathrm{GeV}$ (Ackermann et al. 2016a). We further restrict our attention to objects that also appear in 3LAC (Ackermann et al. 2015b) and have a measured redshift, yielding 122 objects. These are dominated by BL Lac-like (BLL) objects, as opposed to the flat-spectrum radio quasars that compose the vast majority of the remainder of the Fermi AGN population. This is consistent with the strong correlation between AGN type and spectral hardness, which is the reason why BLLs typically dominate at high energies.

The Fermi PSF provides a direct lower limit on the size of any detectable ICC halo, both by smearing the anisotropic structure and through contamination from the much brighter direct emission from the source. For $z \geqslant 1$, the angular size of the region over which pairs are efficiently produced, i.e., the angular size of $D_{\mathrm{pp}}$, is close to the Fermi PSF. A more stringent limit comes from the typical deflections in a large-scale IGMF:

$$
\Delta \alpha_{\mathrm{def}} \approx \frac{\omega_{B} t_{\mathrm{IC}}}{\gamma^{2}} \approx 1.5\left(\frac{B}{10^{-16} \mathrm{G}}\right)\left(\frac{E_{\mathrm{IC}}}{10 \mathrm{GeV}}\right)^{-1}(1+z)^{-4},
$$

where $\quad \omega_{B} \equiv e B / m_{\mathrm{e}} c$ and $t_{\mathrm{IC}} \equiv 3 m_{\mathrm{e}} c / 4 \sigma_{T} u_{s} \approx 2.4 \times$ $10^{12}(1+z)^{-4}$ years, which sets the size of the ICC halos generated by the emission beamed toward us. Noting that the IGMF strength today is redshifted relative to that at high $z$, for a fixed current IGMF magnitude, $\Delta \alpha_{\text {def }} \propto(1+z)^{-2}$ (see Section 3.4). Thus, for a current $10^{-16} \mathrm{G}$ IGMF, when $z>0.6$, the halo size is typically smaller than 0.6 and hence likely to be confused with the central source. This is a moderately strong function of the IGMF strength and ICC halo gamma-ray energy, growing to $z \lesssim 4$ for a present-day $10^{-15} \mathrm{G}$ IGMF or $1 \mathrm{GeV}$ ICC halo photons. However, in those cases, the halo size is limited by the jet width (Section 3.3). For this reason, we impose a limit of $z<0.5$, yielding a set of 84 sources.

The above comprise the intrinsic source selection criteria: appearance in 2FHL and 3LAC with source identification and known redshifts.

\subsection{Intrinsic SEDs}

Neither the 3FGL (the parent catalog of the 3LAC; Acero et al. 2015) nor 2FHL tabulated SEDs are good estimators of the intrinsic $\mathrm{TeV}$ brightness for at least two reasons. First, the curvature in the SED within the energy bands for which the photon spectral indexes are reported in 3FGL $(100 \mathrm{MeV}-$ $100 \mathrm{GeV})$ and $2 \mathrm{FHL}(50 \mathrm{GeV}-2 \mathrm{TeV})$ makes any extrapolation to the VHEGR band of interest, $1 \mathrm{TeV}-10 \mathrm{TeV}$, highly uncertain. Second, absorption on the EBL for sources with 
Table 1

Optimized Source List for a Large-scale, Uniform IGMF

\begin{tabular}{|c|c|c|c|c|c|c|c|c|c|c|c|}
\hline 3FGL Name & Common Names & $\begin{array}{c}E_{p}{ }^{\mathrm{a}} \\
(\mathrm{GeV})\end{array}$ & $\Gamma_{l}^{\mathrm{b}}$ & $\Gamma_{h}{ }^{\mathrm{c}}$ & $z$ & $\begin{array}{l}R_{\text {img }}{ }^{\mathrm{d}} \\
(\mathrm{deg})\end{array}$ & $\begin{array}{c}N_{\mathrm{ph}, \mathrm{f}}^{\mathrm{e}} \\
(\mathrm{ph})\end{array}$ & $\begin{array}{c}\mathcal{B}_{\mathrm{f}}{ }^{\mathrm{f}} \\
\left(\mathrm{ph} / \operatorname{deg}^{2}\right)\end{array}$ & $\begin{array}{c}N_{\mathrm{ph}, \mathrm{b}}^{\mathrm{g}} \\
(\mathrm{ph})\end{array}$ & $\begin{array}{c}\mathcal{B}_{\mathrm{b}}{ }^{\mathrm{h}} \\
\left(\mathrm{ph} / \operatorname{deg}^{2}\right)\end{array}$ & $\begin{array}{l}B_{0}{ }^{\mathrm{i}} \\
(\mathrm{G})\end{array}$ \\
\hline 3FGL J1104.4+3812 & Mkn 421 & 95.38 & 1.77 & $2.14_{-0.10}^{+0.11}$ & 0.03 & 2.0 & 4999 & 43.42 & 4746 & 75.37 & $>10^{-17}$ \\
\hline 3FGL J2347.0+5142 & $\cdots$ & 1.81 & 1.69 & $1.90_{-0.04}^{+0.05}$ & 0.044 & 2.5 & 3176 & 144.57 & 2900 & 133.47 & $\ldots$ \\
\hline 3FGL J1653.9+3945 & Mkn 501 & 236.30 & 1.72 & $2.72_{-0.37}^{+0.51}$ & 0.034 & 1.8 & 2028 & 56.06 & 1990 & 74.14 & $\cdots$ \\
\hline 3 FGL J2000.0+6509 & $\cdots$ & 658.00 & 1.87 & $4.25_{-1.60}^{+1.58}$ & 0.047 & 3.5 & 6610 & 142.40 & 6257 & 135.76 & $\cdots$ \\
\hline 3FGL J1015.0+4925 & $\cdots$ & 2.71 & 1.75 & $1.93_{-0.03}^{+0.03}$ & 0.212 & 2.5 & 1797 & 34.97 & 1756 & 38.88 & $>10^{-16}$ \\
\hline 3FGL J1444.0-3907 & $\cdots$ & 11.9 & 1.67 & $2.14_{-0.09}^{+0.13}$ & 0.065 & 2.5 & 2908 & 124.25 & 2781 & 118.81 & $\cdots$ \\
\hline 3 FGL J0650.7+2503 & $\cdots$ & 281.60 & 1.67 & $2.29_{-1.35}^{+1.64}$ & 0.203 & 2.5 & 2142 & 92.84 & 2069 & 90.54 & $\cdots$ \\
\hline 3FGL J1120.8+4212 & $\ldots$ & 28.65 & 1.56 & $2.15_{-0.19}^{+0.29}$ & 0.124 & 3.0 & 1123 & 34.57 & 1166 & 35.49 & $\cdots$ \\
\hline 3 FGL J1442.8+1200 & $\cdots$ & 0.49 & 2.69 & $1.81_{-0.07}^{+0.08}$ & 0.163 & 2.5 & 921 & 41.95 & 998 & 47.33 & $\cdots$ \\
\hline 3FGL J0508.0+6736 & $\cdots$ & 2.32 & 1.81 & $1.43_{-0.03}^{+0.03}$ & 0.34 & 2.0 & 1927 & 129.53 & 1817 & 129.53 & $\cdots$ \\
\hline 3FGL J0303.4-2407 & $\cdots$ & 1.02 & 1.78 & $1.97_{-0.03}^{+0.03}$ & 0.26 & 2.0 & 984 & 30.18 & 1000 & 41.48 & $>10^{-15}$ \\
\hline 3FGL J0543.9-5531 & $\cdots$ & 0.84 & 0.46 & $1.77_{-0.04}^{+0.05}$ & 0.273 & 2.0 & 1051 & 73.31 & 1039 & 70.55 & $\cdots$ \\
\hline 3FGL J1436.8+5639 & $\cdots$ & 1.05 & 2.54 & $1.79_{-0.09}^{+0.10}$ & 0.15 & 2.0 & 599 & 39.55 & 559 & 40.65 & $\cdots$ \\
\hline 3FGL J2329.2+3754 & $\cdots$ & 2.19 & 2.19 & $1.81_{-0.08}^{+0.11}$ & 0.264 & 2.0 & 1194 & 83.78 & 1217 & 92.32 & $\cdots$ \\
\hline 3FGL J0958.6+6534 & $\cdots$ & 46.64 & 2.35 & $1.35_{-0.34}^{+0.68}$ & 0.367 & 3.0 & 2317 & 62.36 & 2118 & 60.70 & $\cdots$ \\
\hline 3FGL J0449.4-4350 & $\cdots$ & 23.03 & 1.81 & $2.30_{-0.10}^{+0.11}$ & 0.205 & 2.0 & 1653 & 38.17 & 1532 & 41.48 & $>10^{-14}$ \\
\hline 3FGL J0757.0+0956 & $\cdots$ & 59.98 & 2.25 & $1.62_{-0.61}^{+0.93}$ & 0.27 & 2.0 & 775 & 46.71 & 740 & 47.40 & $\cdots$ \\
\hline 3FGL J0622.4-2606 & $\cdots$ & 7.75 & 2.12 & $1.63_{-0.11}^{+0.14}$ & 0.414 & 2.0 & 976 & 57.74 & 1001 & 62.28 & $\ldots$ \\
\hline
\end{tabular}

Notes.

${ }^{\mathrm{a}}$ Energy of power spectrum break in GeV.

${ }^{b}$ Low-energy photon spectral index.

${ }^{c}$ High-energy photon spectral index with $1 \sigma$ error.

d Radius of image selected in degrees.

e Number of front-converted photons within the radius selected.

${ }^{\mathrm{f}}$ Estimate of number of front-converted photons per degree squared from background sources.

$\mathrm{g}$ Number of back-converted photons within radius selected.

h Estimate of number of back-converted photons per degree squared from background sources.

${ }^{\mathrm{i}}$ Values of present-day magnetic field for which the source is in the optimized source list for a large-scale, uniform IGMF (see Section 4.6).

$z>0.1$ can have a substantial impact on the SED above $100 \mathrm{GeV}$, rendering the observed VHEGR SEDs poor estimators of the intrinsic VHEGR SEDs.

For a handful of sources, there exist reported deabsorbed SEDs from air Cherenkov telescopes (e.g., MAGIC, VERITAS, H.E.S.S.). However, these suffer from a number of additional limitations. Typically, they provide measurements over a very limited temporal window of the highly variable emission from gamma-ray bright blazars, and hence are often poor estimators of the time-averaged fluence over long periods. Additionally, the deabsorption prescriptions vary substantially among sources and thus they do not provide a homogeneous class of SED estimates. Finally, for all but the brightest sources, the reported Cherenkov telescope SEDs are limited to below the VHEGR energy band of interest, producing the same uncertainties that arise from 2FHL and 3FGL. They do, however, indicate the degree of variability we may expect over decadal timescales.

Therefore, we independently generate composite SEDs by collating the 3FGL and 2FHL band-specific energy flux measurements for each of the 84 common sources with redshifts below 0.5 as described in Appendix E. That is, gamma-ray SEDs were produced by compiling the $0.1-0.3 \mathrm{GeV}, \quad 0.3-1 \mathrm{GeV}, \quad 1-3 \mathrm{GeV}, \quad 3-10 \mathrm{GeV}, \quad$ and $10-100 \mathrm{GeV}$ flux measurements reported in 3FGL and $50-171 \mathrm{GeV}, 171-585 \mathrm{GeV}$, and $585-2000 \mathrm{GeV}$ reported in 2FHL. The former (3FGL) are averaged over four years (2008
August-2012 July) and the latter (2FHL) are averaged over seven years (2008 August-2015 August), and thus present nearly contemporaneous ranges extending over many years.

These were deabsorbed using the pair-creation optical depth given by

$$
\tau_{\mathrm{obs}}(E, z)=\int_{0}^{z} d z^{\prime} \frac{d D_{P} / d z}{D_{\mathrm{pp}}[E(1+z), z]},
$$

where $D_{P}$ is the proper distance to the source, evaluated at the geometric center of the energy bin, i.e., we set

$$
F_{E, \text { deabs }}=F_{E, \text { obs }} e^{\tau_{\mathrm{obs}}(E, z)},
$$

where $F_{E \text {,obs }}$ is the specific energy flux. Both the observed and deabsorbed SEDs for Mkn 421 are shown in Figure 3, with the remainder of the sources used here (i.e., those listed in Table 1) shown in Appendix E.

The deabsorbed SEDs are generally well-fit by a broken power-law SED, defined by a normalization, a pivot energy $E_{p}$, and photon spectral indexes above and below $E_{p}, \Gamma_{h}$ and $\Gamma_{l}$, respectively:

$$
\frac{d N}{d E}=f_{0} \begin{cases}\left(E / E_{p}\right)^{-\Gamma_{l}} & E \leqslant E_{p} \\ \left(E / E_{p}\right)^{-\Gamma_{h}} & E>E_{p} .\end{cases}
$$

A maximum-likelihood fit of the broken power-law SED model was performed for each candidate source. The result is also 


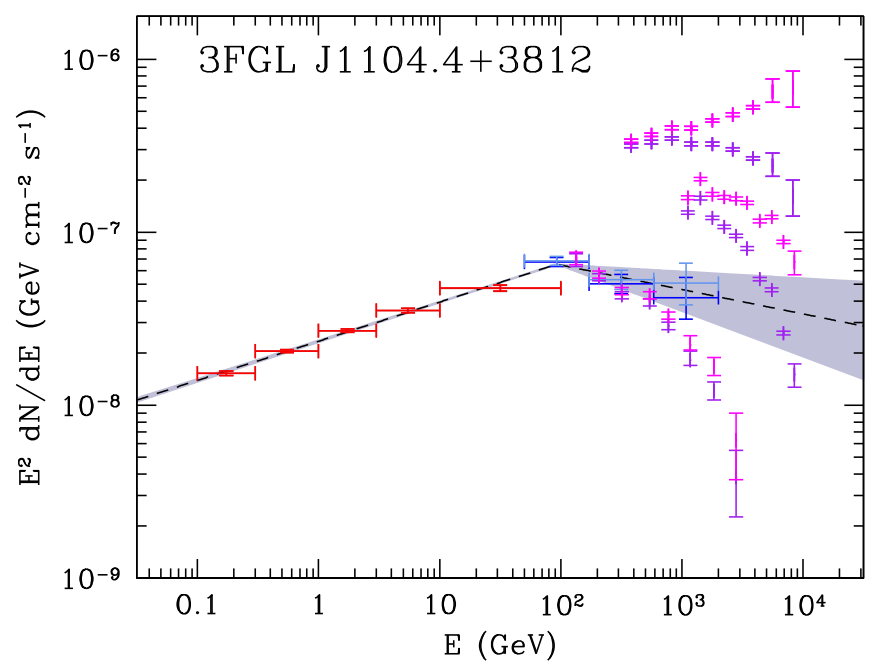

Figure 3. Composite SED for Mkn 421 taken from the 3FGL (red) and 2FHL (dark blue) catalogs and their deabsorbed counterparts (orange and light blue, respectively, though the former values are indistinguishable from the observed ones). A broken power-law fit is shown by the dashed line; the gray band denotes the uncertainty in the SED reconstruction. For comparison, VHEGR observations by ground-based air Cherenkov telescopes (Whipple observations of the high state in 2000/2001, H.E.S.S. observations in 2004, and MAGIC observations in 2004/2005, top to bottom, adapted from Figure 10 of Albert et al. 2007) are shown in purple (observed) and magenta (deabsorbed).

shown by the dashed line in Figure 3 for Mkn 421. We visually verified that small variations in the assumed initial starting point results in negligible variations in the final fit parameter values (though large variations can result in erroneous fit results).

Two classes of qualitatively different SEDs were found from the deabsorbed spectral fits: sources with spectral breaks that are convex $\left(\Gamma_{h}>\Gamma_{l}\right)$ and those that are concave $\left(\Gamma_{h}<\Gamma_{l}\right)$. The former are consistent with the expectation from single-zone IC models of VHEGR sources (Ghisellini et al. 1998; Abdo et al. 2010c; Ackermann et al. 2016a). The latter suggest the need for an additional spectral component, either due to an additional Comptonizing population or alternative emission source (Böttcher et al. 2013; Cerruti et al. 2015; Zacharias \& Wagner 2016).

Uncertainties on the fit parameters were obtained via a Monte Carlo analysis. Trial fluctuations in the fit parameters were constructed from normal distributions with standard deviations given by the Fisher matrix error estimates. An estimate of the allowed range was obtained by taking the collection of parameter values for which the log-likelihood, i.e., $\chi^{2}$, increased by unity, shown by the gray bands in Figure 3 . This was especially important for sources with only upper limits at high energies, and thus for which the uncertainty in the high-energy SEDs was highly asymmetric.

Generally, the normalization, $E_{p}$, and $\Gamma_{l}$ were tightly constrained; this is in part a selection effect as each object is a well-characterized Fermi source. Thus, in our set of bright, nearby gamma-ray bright AGNs, we fix these to their observed (normalization) or fitted $\left(E_{p}\right.$ and $\left.\Gamma_{l}\right)$ values. In contrast, $\Gamma_{h}$ is considerably more uncertain, often as a result of a high $E_{p}$ and larger uncertainties or upper limits on the intrinsic high-energy flux estimates. Therefore, for the purpose of generating ICC halo realizations, we stochastically choose $\Gamma_{h}$ over the permitted range. Because this is typically asymmetric, permitting either much smaller or larger values of $\Gamma_{h}$, we assume that the probability of a given $\Gamma_{h}$ is well approximated by two onesided normal distributions centered on the best-fit value with standard deviations set by the range obtained by the Monte Carlo procedure above and below.

The key intrinsic target parameters that enter the generation of mock realizations of the sample are the number of observed source photons, $z$, and the source intrinsic SED fit parameters. These are listed in Table 1 for the Fermi targets that are used in this paper (see also Section 4.6).

\subsection{Local Gamma-Ray Neighborhood}

In addition to the intrinsic source requirements, the diffuse, large-scale nature of the ICC halos places constraints on the neighborhood of targets. While these are essentially limits on potential contaminating features, for the purpose of target selection, this reduces to limits on neighboring sources and large-scale background gradients.

Even weak neighboring sources can produce a large bias in the angular power spectra. While we defer a characterization of this signal to Section 4.4.2, an initial target-list cut was made to remove all sources with bright neighbors within $2^{\circ}$. Beyond $2^{\circ}$, neighbors were permitted, though the area over which the power spectrum analysis was performed was restricted to prevent contamination. This is illustrated in Figure 4, which shows examples of excluded, restricted, and ideal sources.

The gamma-ray background varies substantially from source to source as a result of the different sky locations. This is dominated by the diffuse Galactic component and becomes noticeably worse at low Galactic latitudes, where it imparts substantial gradients to the gamma-ray counts. Rather than attempting to model this component, we exclude sources with strong background gradients visible over scales of $10^{\circ}$ (typically corresponding to $|l|<18^{\circ}$ ) and make an estimate of the background photon density for each target source individually. In practice, the presence of a background gradient appears dominantly in the dipolar power, and thus is distinguishable from the bipolar signals of interest (see Section 4.4.2).

Therefore, the above comprise the extrinsic source selection criteria: no neighbors within $2^{\circ}$, restricting from $4^{\circ}$ as necessary, and no large-scale gradients in the background flux. The key extrinsic target parameters that enter the generation of mock realizations of the sample are the maximum noncontaminated angular radius, $R_{\max }$, and the total number of front- and back-converted photons within the permitted region due to the source and background. There are 27 objects that are sufficiently isolated and satisfy the intrinsic source parameter selection criteria, which are shown in Table 2. The final selection of the Fermi targets listed in Table 1 will be described in Section 4.6.

\subsection{Jet Opening and Viewing Angles}

For most Fermi blazars, the viewing and intrinsic opening angles are poorly constrained. However, the subset of objects that also appear in the sample monitored by the MOJAVE group provides some guidance on the parsec-scale radio opening and viewing angles (Pushkarev et al. 2009). Generally, Fermi blazars exhibit radio jets that are intrinsically narrower 

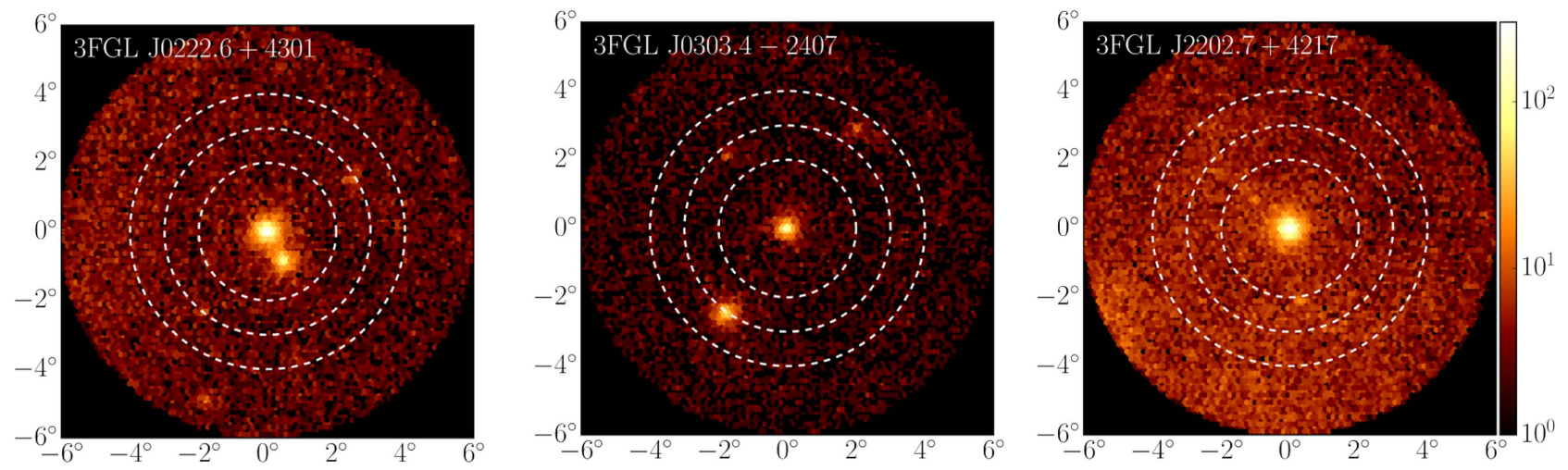

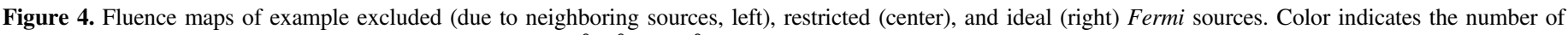
photons within a given pixel. Dashed circles have radii of $2^{\circ}, 3^{\circ}$, and $4^{\circ}$.

than the gamma-ray dim population. The distribution peaks near a median of 1.0 and is well-fit by a generalized $\lambda$ distribution with parameters given in Appendix A (Pushkarev et al. 2009).

Determining the gamma-ray jet opening angles is complicated for a number of reasons. First, obtaining the radio opening angles is itself challenging. While measuring the apparent (projected) opening angle is straightforward, determining the intrinsic opening angle typically requires kinematic information obtained from multiple widely separated epochs of imaging observations. Second, the radio and gamma-ray opening angles need not be the same and are generally different. Moreover, the current uncertainty in the gamma-ray emission mechanism precludes using spectral information to relate the two directly.

To assess the relationship between the two, we begin with the following assumptions:

1. The flux distribution of Fermi sources is limited from above, i.e., they exhibit a maximum intrinsic luminosity.

2. The intrinsic opening angles of the gamma-ray and radio jets are proportional, i.e., broad radio jets are broad gamma-ray jets and vice versa.

3. The gamma-ray jet is structured as a Gaussian with a source-dependent standard deviations $\theta_{\text {jet }}$. That is, the gamma-ray flux within the jet observed at an angle $\Theta$ is given by

$$
\mathcal{F}=\mathcal{F}_{0} e^{-\Theta^{2} / 2 \theta_{\text {jet. }}^{2}}
$$

The first is a good approximation in practice since Fermi only sees the bright end of the hard gamma-ray blazar luminosity function. The second is natural given that the overwhelming majority of gamma-ray bright AGNs are blazars (Ackermann et al. 2011, 2015b). The quantitative consequence is that the gamma-ray jet scale, $\theta_{\text {jet }}$, is related to the intrinsic radio jet FWHM, $\alpha_{\text {int }}$, by some proportionality constant

$$
\theta_{\text {jet }}=\sigma_{\text {jet }} \alpha_{\text {int }} .
$$

The third is already made in the gamma-ray halo models described in Broderick et al. (2016) and employed here. Importantly, note that the assumed structure is effectively a condition on the apparent jet opening angle, $\alpha_{\text {app }}$; although generally $\tan \alpha_{\text {app }}=\tan \alpha_{\text {int }} / \sin \Theta$, for small radio opening and viewing angles, this is approximately $\tan \alpha_{\mathrm{app}} \approx \alpha_{\mathrm{int}} / \Theta$, and hence the condition imposed by the third can be cast in terms of the apparent opening angle alone:

$$
\mathcal{F}=\mathcal{F}_{0} e^{-1 / 2 \sigma_{\text {jet }}^{2} \tan ^{2} \alpha_{\text {app }}} .
$$

This has two consequences. First, the number of Fermi blazars for which $\alpha_{\text {app }}$ is known is comparatively large. Second, because $\alpha_{\text {app }}$ is directly measured during a single epoch of radio observations, it is much better known than $\alpha_{\text {int }}$ or $\Theta$.

While the value of $\mathcal{F}_{0}$ is unknown for any given source, the presence of an upper limit implies that Fermi sources should populate the region in the $\mathcal{F}$ - $\alpha_{\text {app }}^{-1}$ plane defined by some $\mathcal{F}_{0 \text {,max }}$. Shown in Figure 5, this is the clearly the case-sources with large $\alpha_{\text {app }}^{-1}$ have systematically lower fluxes, falling in a manner consistent with the Gaussian dependence posited. This presents a direct way in which to measure $\sigma_{\text {jet }}$ by fitting the envelope of points in the $\mathcal{F}-\alpha_{\text {app }}^{1}$ plane.

Interestingly, the value obtained depends strongly on spectral hardness. Softer sources $\left(\Gamma_{l}>2.1\right)$ have systematically broader gamma-ray jets, with $\sigma_{\text {jet }} \approx 2.5$; the hard sources of interest here $\left(\Gamma_{h}<2.1\right)$ have $45 \%$ narrower jets, with $\sigma_{\text {jet }} \approx 1.6$. It is this latter relationship we adopt.

Note that this implies that the gamma-ray emission is beamed over a substantially larger angle than subtended by the parsec-scale radio jet. The ratio of the FWHMs of the gammaray and parsec-scale radio jets is $(8 \ln 2)^{1 / 2} \sigma_{\text {jet }}=3.77$, diffusing the emission over a solid-angle nearly 14 times larger. This suggests that the beaming of the gamma-ray emission is similar, qualitatively, to the presumed jet structure near its base, i.e., within the collimating region.

This does not mean, however, that we anticipate large beaming corrections to the apparent gamma-ray flux or that there should be a large population of non-blazar gamma-ray sources observed by Fermi. Generally, parsec-scale jets are more tightly collimated in comparison to the typical beaming angle of the radio emission, with

$$
\alpha_{\mathrm{int}} \approx \frac{0.3}{\Gamma_{\mathrm{jet}}},
$$

where $\Gamma_{\text {jet }}$ is the jet Lorentz factor (not to be confused with a photon spectral index); this continues to hold for the Fermi subset of MOJAVE sources (Pushkarev et al. 2009). That is, the typical angular scale over which the radio emission is beamed, $\Gamma_{\text {jet }}^{-1}$, is itself three times the parsec-scale jet FHWM. Comparing the gamma-ray and radio beaming angle gives 
Table 2

List of Source

\begin{tabular}{|c|c|c|c|c|c|c|c|c|c|c|}
\hline \multicolumn{3}{|c|}{ Names } & \multirow[b]{2}{*}{$z^{\mathrm{a}}$} & \multicolumn{2}{|c|}{ Reported Fluxes } & \multicolumn{5}{|c|}{ SED Fit Results } \\
\hline $3 \mathrm{FGL}$ & 2FHL & $\mathrm{TeV}$ & & $\begin{array}{c}F_{3 \mathrm{FGL}}{ }^{\mathrm{b}} \\
\left(\mathrm{ph} \mathrm{cm}^{-2} \mathrm{~s}^{-1}\right)\end{array}$ & $\begin{array}{c}F_{2 \mathrm{FHL}}{ }^{\mathrm{c}} \\
\left(\mathrm{ph} \mathrm{cm}^{-2} \mathrm{~s}^{-1}\right)\end{array}$ & $\begin{array}{c}F_{\mathrm{TeV}^{\mathrm{d}}} \\
\left(\mathrm{GeV} \mathrm{cm}^{-2} \mathrm{~s}^{-1}\right)\end{array}$ & $\begin{array}{c}f_{0} \\
\left(\mathrm{ph} \mathrm{cm}^{-2} \mathrm{~s}^{-1}\right)\end{array}$ & $\begin{array}{c}E_{p} \\
(\mathrm{GeV})\end{array}$ & $\Gamma_{l}$ & $\Gamma_{h}{ }^{\mathrm{e}}$ \\
\hline $\mathrm{J} 1104.4+3812$ & $\mathrm{~J} 1104.4+3812$ & $\mathrm{~J} 1104+3811$ & 0.031 & $3.03 \times 10^{-8}$ & $1.24 \times 10^{-9}$ & $4.69_{-1.04}^{+1.20} \times 10^{-8}$ & $6.53 \times 10^{-8}$ & 95.38 & 1.77 & $2.14_{-0.10}^{+0.11}$ \\
\hline$J 1653.9+3945$ & J1653.9+3945 & $\mathrm{J} 1653+3945$ & 0.0337 & $9.74 \times 10^{-9}$ & $4.78 \times 10^{-10}$ & $1.14_{-0.58}^{+0.79} \times 10^{-8}$ & $3.21 \times 10^{-8}$ & 236.30 & 1.72 & $2.72_{-0.37}^{+0.51}$ \\
\hline J0449.4-4350 & J0449.4-4349 & J0449-4350 & 0.205 & $1.03 \times 10^{-8}$ & $1.65 \times 10^{-10}$ & $5.07_{-1.75}^{+2.14} \times 10^{-9}$ & $1.58 \times 10^{-8}$ & 23.03 & 1.81 & $2.30_{-0.10}^{+0.11}$ \\
\hline $\mathrm{J} 0508.0+6736$ & J0507.9+6737 & $\mathrm{J} 0507+6737$ & 0.34 & $2.12 \times 10^{-9}$ & $1.63 \times 10^{-10}$ & $4.09_{-0.70}^{+0.74} \times 10^{-8}$ & $1.32 \times 10^{-9}$ & 2.32 & 1.81 & $1.43_{-0.03}^{+0.03}$ \\
\hline $\mathrm{J} 1015.0+4925$ & $\mathrm{~J} 1015.0+4926$ & $\mathrm{~J} 1015+4926$ & 0.212 & $7.35 \times 10^{-9}$ & $1.62 \times 10^{-10}$ & $1.19_{-0.21}^{+0.23} \times 10^{-8}$ & $7.89 \times 10^{-9}$ & 2.71 & 1.75 & $1.93_{-0.03}^{+0.03}$ \\
\hline $\mathrm{J} 2000.0+6509$ & $\mathrm{~J} 2000.1+6508$ & $\mathrm{~J} 1959+6508$ & 0.047 & $5.84 \times 10^{-9}$ & $1.57 \times 10^{-10}$ & $4.49_{-3.45}^{+4.91} \times 10^{-9}$ & $1.15 \times 10^{-8}$ & 658.00 & 1.87 & $4.25_{-1.60}^{+1.58}$ \\
\hline $\mathrm{J} 0650.7+2503$ & $\mathrm{~J} 0650.7+2502$ & $\mathrm{~J} 0650+2503$ & 0.203 & $2.16 \times 10^{-9}$ & $1.26 \times 10^{-10}$ & $6.10_{-5.25}^{+20.21} \times 10^{-9}$ & $8.75 \times 10^{-9}$ & 281.60 & 1.67 & $2.29_{-1.35}^{+1.64}$ \\
\hline J2009.3-4849 & J2009.4-4849 & J2009-4849 & 0.071 & $3.55 \times 10^{-9}$ & $9.70 \times 10^{-11}$ & $1.15_{-0.92}^{+1.89} \times 10^{-9}$ & $7.08 \times 10^{-9}$ & 76.45 & 1.77 & $2.71_{-0.39}^{+0.64}$ \\
\hline J0303.4-2407 & J0303.3-2407 & $\mathrm{J} 0303-2407$ & 0.26 & $5.74 \times 10^{-9}$ & $8.62 \times 10^{-11}$ & $7.03_{-1.28}^{+1.44} \times 10^{-9}$ & $5.68 \times 10^{-9}$ & 1.02 & 1.78 & $1.97_{-0.03}^{+0.03}$ \\
\hline J1444.0-3907 & J1443.9-3909 & $\cdots$ & 0.0654 & $3.23 \times 10^{-9}$ & $8.22 \times 10^{-11}$ & $2.94_{-1.18}^{+1.44} \times 10^{-9}$ & $5.43 \times 10^{-9}$ & 11.90 & 1.67 & $2.14_{-0.09}^{+0.13}$ \\
\hline $\mathrm{J} 2347.0+5142$ & $\mathrm{~J} 2347.1+5142$ & $\mathrm{~J} 2346+5142$ & 0.044 & $2.42 \times 10^{-9}$ & $7.48 \times 10^{-11}$ & $4.47_{-1.21}^{+1.39} \times 10^{-9}$ & $2.43 \times 10^{-9}$ & 1.81 & 1.69 & $1.90_{-0.04}^{+0.05}$ \\
\hline J0648.8+1516 & J0648.6+1516 & $\mathrm{J} 0648+1516$ & 0.179 & $1.45 \times 10^{-9}$ & $5.62 \times 10^{-11}$ & $4.49_{-1.36}^{+1.74} \times 10^{-9}$ & $1.21 \times 10^{-9}$ & 1.03 & 0.97 & $1.81_{-0.06}^{+0.05}$ \\
\hline J0543.9-5531 & J0543.9-5533 & $\cdots$ & 0.273 & $1.61 \times 10^{-9}$ & $5.27 \times 10^{-11}$ & $5.90_{-1.64}^{+1.88} \times 10^{-9}$ & $1.17 \times 10^{-9}$ & 0.84 & 0.46 & $1.77_{-0.04}^{+0.05}$ \\
\hline $\mathrm{J} 1512.8-0906$ & $\mathrm{~J} 1512.7-0906$ & J1512-0906 & 0.36 & $4.11 \times 10^{-8}$ & $4.59 \times 10^{-11}$ & $1.71_{-0.21}^{+0.27} \times 10^{-9}$ & $7.24 \times 10^{-8}$ & 1.27 & 2.22 & $2.56_{-0.02}^{+0.02}$ \\
\hline $\mathrm{J} 1120.8+4212$ & $\mathrm{~J} 1120.8+4212$ & $\cdots$ & 0.124 & $1.16 \times 10^{-9}$ & $4.18 \times 10^{-11}$ & $1.70_{-1.07}^{+1.61} \times 10^{-9}$ & $2.92 \times 10^{-9}$ & 28.65 & 1.56 & $2.15_{-0.19}^{+0.29}$ \\
\hline $\mathrm{J} 1117.0+2014$ & $\mathrm{~J} 1116.9+2014$ & $\ldots$ & 0.138 & $1.74 \times 10^{-9}$ & $3.85 \times 10^{-11}$ & $2.53_{-1.03}^{+1.33} \times 10^{-9}$ & $1.89 \times 10^{-9}$ & 3.02 & 1.77 & $1.95_{-0.08}^{+0.09}$ \\
\hline $\mathrm{J} 2250.1+3825$ & $\mathrm{~J} 2249.9+3826$ & $\mathrm{~J} 2250+3824$ & 0.119 & $1.10 \times 10^{-9}$ & $3.38 \times 10^{-11}$ & $1.91_{-0.66}^{+0.84} \times 10^{-9}$ & $8.83 \times 10^{-10}$ & 0.46 & 2.72 & $1.90_{-0.05}^{+0.06}$ \\
\hline J0627.0-3529 & J0626.9-3528 & $\cdots$ & 0.05494 & $1.43 \times 10^{-9}$ & $2.92 \times 10^{-11}$ & $2.85_{-0.80}^{+1.12} \times 10^{-9}$ & $1.01 \times 10^{-9}$ & 0.37 & 2.77 & $1.87_{-0.05}^{+0.04}$ \\
\hline J0622.4-2606 & J0622.4-2604 & $\ldots$ & 0.41449 & $1.14 \times 10^{-9}$ & $2.58 \times 10^{-11}$ & $5.41_{-2.69}^{+3.71} \times 10^{-9}$ & $8.75 \times 10^{-10}$ & 7.75 & 2.12 & $1.63_{-0.11}^{+0.14}$ \\
\hline $\mathrm{J} 2131.5-0915$ & J2131.4-0914 & $\cdots$ & 0.449 & $8.63 \times 10^{-10}$ & $2.49 \times 10^{-11}$ & $4.21_{-2.08}^{+3.26} \times 10^{-9}$ & $6.00 \times 10^{-10}$ & 4.26 & 2.27 & $1.64_{-0.12}^{+0.12}$ \\
\hline J2016.4-0905 & J2016.5-0904 & $\cdots$ & 0.367 & $1.40 \times 10^{-9}$ & $1.86 \times 10^{-11}$ & $1.63_{-0.74}^{+1.00} \times 10^{-9}$ & $1.50 \times 10^{-9}$ & 2.37 & 1.89 & $1.99_{-0.11}^{+0.11}$ \\
\hline $\mathrm{J} 0115.8+2519$ & $\mathrm{~J} 0115.8+2519$ & $\cdots$ & 0.358 & $1.15 \times 10^{-9}$ & $1.83 \times 10^{-11}$ & $2.08_{-0.78}^{+1.03} \times 10^{-9}$ & $9.38 \times 10^{-10}$ & 0.80 & 2.54 & $1.89_{-0.06}^{+0.07}$ \\
\hline $\mathrm{J} 0757.0+0956$ & J0756.8+0955 & $\cdots$ & 0.266 & $1.86 \times 10^{-9}$ & $1.73 \times 10^{-11}$ & $2.65_{-2.46}^{+6.98} \times 10^{-9}$ & $8.99 \times 10^{-10}$ & 59.98 & 2.25 & $1.62_{-0.61}^{+0.93}$ \\
\hline J0958.6+6534 & J0958.3+6535 & $\ldots$ & 0.367 & $1.38 \times 10^{-9}$ & $1.54 \times 10^{-11}$ & $4.25_{-3.71}^{+6.44} \times 10^{-9}$ & $5.73 \times 10^{-10}$ & 46.64 & 2.35 & $1.35_{-0.34}^{+0.68}$ \\
\hline $\mathrm{J} 2329.2+3754$ & $\mathrm{~J} 2329.2+3754$ & $\cdots$ & 0.264 & $8.40 \times 10^{-10}$ & $1.43 \times 10^{-11}$ & $1.99_{-0.95}^{+1.25} \times 10^{-9}$ & $6.34 \times 10^{-10}$ & 2.19 & 2.19 & $1.81_{-0.08}^{+0.11}$ \\
\hline $\mathrm{J} 1436.8+5639$ & $\mathrm{~J} 1437.0+5639$ & $\cdots$ & 0.15 & $4.71 \times 10^{-10}$ & $1.40 \times 10^{-11}$ & $1.39_{-0.68}^{+0.89} \times 10^{-9}$ & $3.18 \times 10^{-10}$ & 1.05 & 2.54 & $1.79_{-0.09}^{+0.10}$ \\
\hline $\mathrm{J} 1442.8+1200$ & $\mathrm{~J} 1442.9+1159$ & $\mathrm{~J} 1442+1200$ & 0.16309 & $5.59 \times 10^{-10}$ & $1.39 \times 10^{-11}$ & $1.54_{-0.67}^{+0.84} \times 10^{-9}$ & $3.57 \times 10^{-10}$ & 0.49 & 2.69 & $1.81_{-0.07}^{+0.08}$ \\
\hline
\end{tabular}

Notes.

a 3FGL value.

b $1-100 \mathrm{GeV}$ fluence from 3FGL.

c $50-2000 \mathrm{GeV}$ fluence from 3FGL.

${ }^{\mathrm{d}} E^{2} d N / d E$ at $(1+z) \mathrm{TeV}$.

${ }^{\mathrm{e}}$ Uncertainty in $\Gamma$ at $(1+z) \mathrm{TeV}$. 


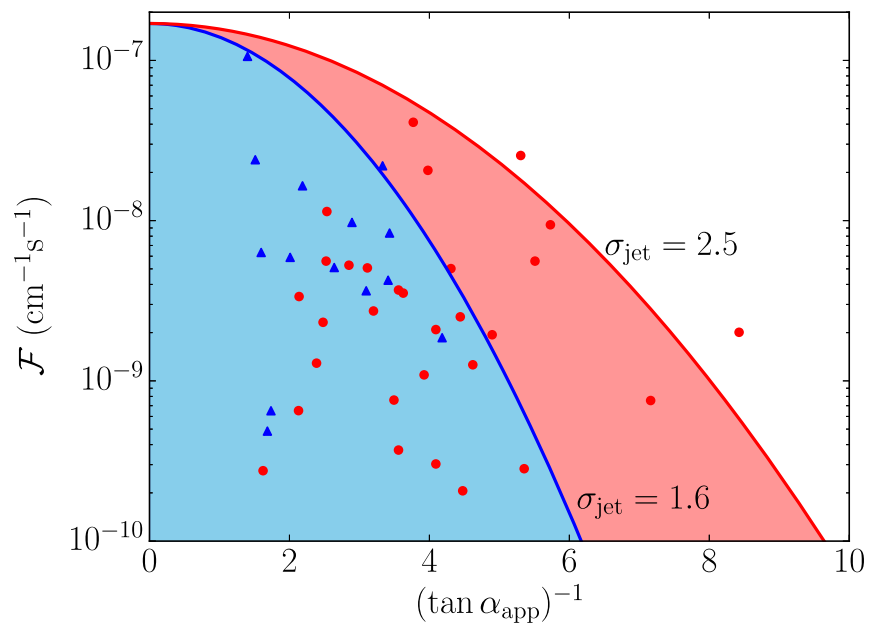

Figure 5. Flux vs. viewing angle in units of radio jet width, approximated by $\left(\tan \alpha_{\text {app }}\right)^{-1}$ where $\alpha_{\text {app }}$ is the apparent parsec-scale radio opening angle for hard $\left(\Gamma_{l}<2.1\right.$, blue $)$ and soft $\left(\Gamma_{l}>2.1\right.$, red) Fermi sources in the MOJAVE database. Fits to the upper envelope assuming a Gaussian jet are shown by the light-blue filled and light-red filled regions.

$\theta_{\text {jet }} \Gamma_{\text {jet }} \approx 0.48$ for the hard gamma-ray blazars. That is, for these sources, the gamma-ray emission is moderately more beamed than the radio emission. Despite having a $\theta_{\text {jet }} \Gamma_{\text {jet }}$ that is roughly $40 \%$ larger, this remains true for the soft gamma-ray blazars - the gamma-ray emission is more beamed than the radio emission.

Because we do not have an a priori estimate of either $\theta_{\text {jet }}$ or $\Theta$ for any particular source, for the purpose of creating ICC halo realizations, we randomly generate values using the following procedure.

1. We select a value of $\alpha_{\text {int }}$ from the distribution of intrinsic opening angles in Pushkarev et al. (2009), as described in Appendix A.

2. Based on $\alpha_{\text {int }}$, we estimate the relativistic beaming angle to be $\Gamma_{\text {jet }}^{-1} \approx \alpha_{\text {int }} / 0.3$. To appear as a blazar, we must be viewing the source within this angle, and thus choose $\Theta$ from an isotropic distribution within $\Gamma_{\text {jet }}^{-1}$.

3. Finally, we obtain the gamma-ray jet half-opening angle from $\theta_{\text {jet }}=\sigma_{\text {jet }} \alpha_{\text {int }}$ with $\sigma_{\text {jet }}=1.6$.

\subsection{IGMF Strength and Orientation}

In the presence of a large-scale IGMF, the ICC halo images depend upon the assumed strength and orientation of the IGMF. Without any prior knowledge of either the strength or orientation of the IGMF, we consider variations in both. In the case of the former, we set the strength of the IGMF today, $B_{0}$, and assign the local strength at the source to be the appropriately redshifted value, i.e., the local magnetic field strength is

$$
B=B_{0}(1+z)^{2} .
$$

For a given analysis, we assume that all sources have the same value of $B_{0}$, assessing the detectability of the ICC halos as a function of field strength. Because we restrict our attention to $z<0.5$, this has at most a factor of two impact on the assumed $B$.

Realizations for the orientation of the field are generated from an isotropic distribution for each image independently.
That is, while in this limit we assert that the field is coherent over scales large in comparison with the gamma-ray jet widths, we permit large variations in the direction of the IGMF across the sky, differing along the lines of sight toward the Fermi AGN in our sample. This is consistent with a picture in which the ICC halos are produced in cosmological voids in which the IGMF has been imprinted from early times. We note that this is rather pessimistic - correlations in the orientations of ICC halos from neighboring VHEGR sources would permit coherent stacking, which we ignore here.

\section{Asymmetric Signatures of Halos}

As described in Section 1, we exploit the anticipated structure in the ICC halos by constructing a statistical measure of the anisotropy. To do this, we focus on the angular power spectra defined in terms of an event-specific polar angle, $\theta_{j}$, about the source center. This is a natural statistic because it is sensitive in particular to anisotropic structure yet independent of the absolute source orientation. Moreover, it is only weakly contaminated by the known systematics in the Fermi LAT instrument responses and the background source population, and in a way that is easily distinguishable from the ICC halo signal of interest. To illustrate this, we begin with an example that makes use of a toy model with many of the key features of the ICC halos we described in Section 2.

A realization of this toy model is shown in Figure 6. This includes equal contributions from a uniform background and from a central source, totaling 4000 photons and comparable to a typical bright Fermi AGN. In addition, there is an anisotropic halo component (red) containing $10 \%$ of the photons in the source drawn from the ad hoc flux distribution indicated by the contours. All components were convolved with a Gaussian PSF with standard deviation 0.2 , comparable to the scale of the Pass 8R2_V6 PSF for front-converted events in the LAT.

In the absence of component color coding, the subdominance of the ICC excess makes it difficult to identify directly from a single source. Typically, this is dealt with by stacking multiple images, increasing the statistical significance with which the halo component can be isolated. We therefore show a stacked image of 18 realizations of the same cartoon halo in Figure 6. Because the orientation of the putative ICC halo feature is also randomly varied (corresponding to different source and IGM orientations) in contrast to the single image, the stacked image exhibits nearly no angular structure. Nevertheless, there is a small gamma-ray excess at large angular scales. In Figure 7, this is shown explicitly in comparison to the case when the halo is absent, beginning near angular scales of $1^{\circ}$ (where the central source ceases to dominate over the background). The interpretation of the excess is complicated, however, by uncertainty in the structure of the large-scale tails of the PSF or the background flux: even marginal modifications of either can absorb the halo signal in its entirety.

Instead, we focus on the anisotropic structure of the ICC halo, which presents a unique signature that is difficult to confuse with instrumental response. Explicitly, we construct an angular power spectrum of the surrounding photon positions about the source, defined by

$$
\mathcal{P}_{m} \equiv \frac{1}{N^{2}}\left|\sum_{j} e^{i m \theta_{j}}\right|^{2},
$$



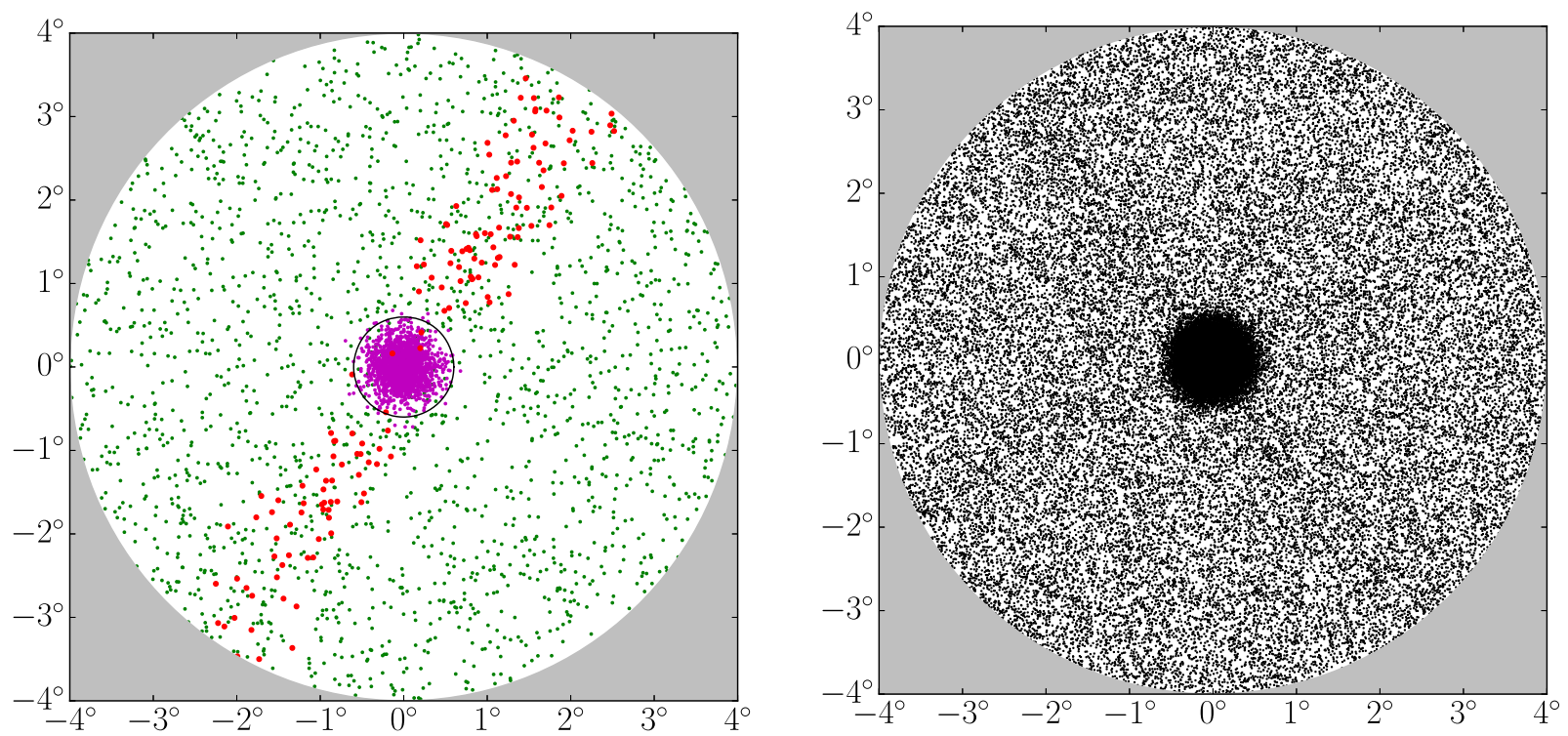

Figure 6. Left: realization of 4000 photons from a toy model that exhibits the anisotropy arising from the mechanisms described in Figure 1. The contributions from the central source, diffuse background, and ICC halo component are shown in purple, green, and red, respectively. The excluded central region is shown in black. Note that despite the clear structure within the ICC halo component, it is strongly subdominant to the otherwise isotropic source and background components (contributing roughly $5 \%$ of the total photons). Right: stacked toy model images of 18 sources with arbitrary ICC halo orientations. At this point, the structure is effectively erased.

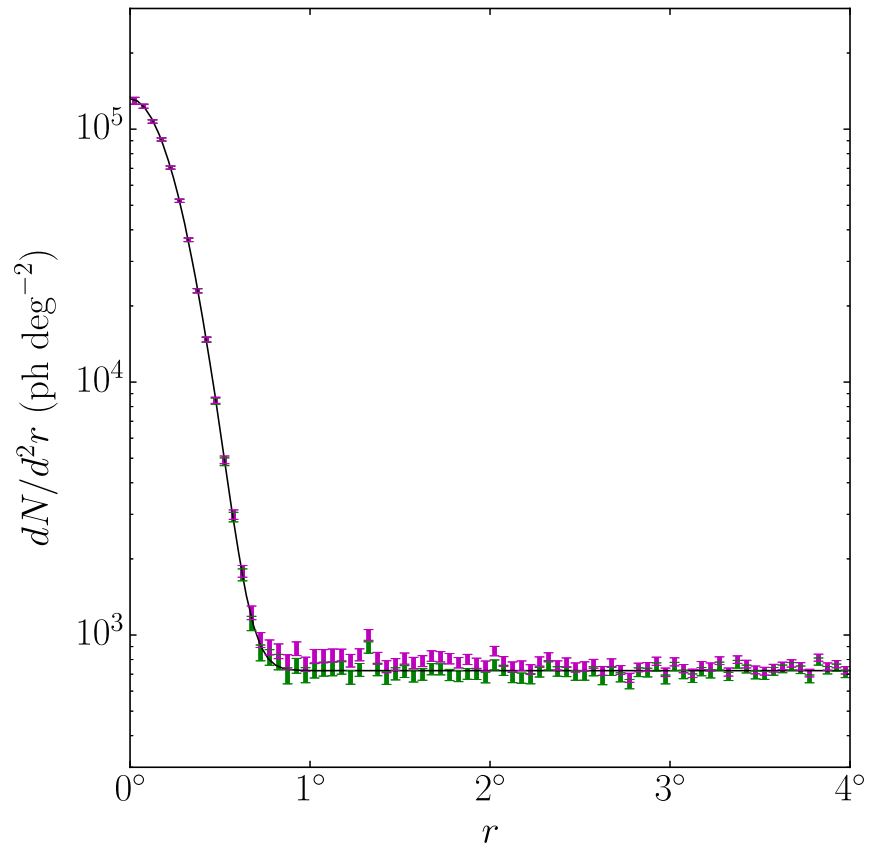

Figure 7. Radial distribution of the gamma-ray surface brightness from the stacked toy model images in Figure 6 with (purple) and without (green) the ICC halo included. This is compared with a model comprised of a central point source and uniform background, convolved with a toy Gaussian PSF with standard deviation 0.2 . While the normalizations for the two components differ slightly from their input values, they are capable of providing a good fit even in the presence of the ICC halos. In any case, any disparity is insufficient to convincingly rule out modifications of the large-angle wings of the PSF.

where $\theta_{j}$ is the polar angle of the $j$ th gamma ray about the image center relative to a fiducial direction and $N$ is the total number of gamma rays. To remove the bulk of the source contribution and eliminate the unresolved structure near the origin, we mask the inner regions prior to constructing $\mathcal{P}_{m}$; for illustrative purposes, here we simply exclude the inner 0.6 , though in practice we implement an energy-dependent mask

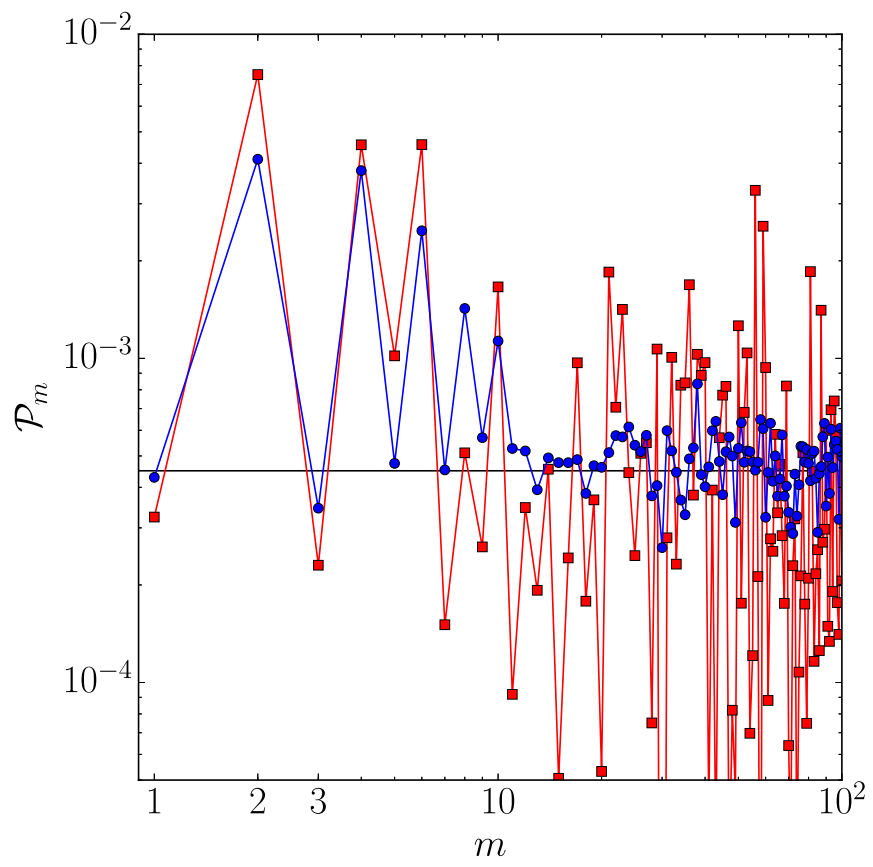

Figure 8. Azimuthal power spectra of a single realization (red squares) and stacked azimuthal power spectra of the 18 realizations (blue circles) of the toy ICC halo model. In these, the anisotropic halo component generates a clear signal in even multipoles, beginning with the quadrupole, due to the underlying symmetry of the ICC halo images. For reference, the Poisson noise limit is show by the black line to which all models asymptote at large $m$.

(see Section 4.3). The ICC halos generate a characteristic power spectrum due to their bimodal structure, shown by the red points in Figure 8, which is dominated by $m=2$ and the even multipoles that follow, and is qualitatively distinct from most potential image contaminants. In contrast, Poisson noise from a cylindrically symmetric source (e.g., the PSF-convolved central AGN or background) is flat, shown by the black line in 
Figure 8 , and therefore easily distinguished from the anisotropic ICC halos.

More importantly, the $\mathcal{P}_{m}$ are independent of the orientation of the halo structure. Thus, it is possible to stack the $\mathcal{P}_{m}$ from many sources directly, improving their estimation and thereby improving the significance with which halos may be detected. For example, the $\mathcal{P}_{m}$ arising from stacking the angular power spectra of the same 18 realizations used to generate Figure 6 is shown explicitly by the blue line in Figure 8 . In this, the halo structure signal at $m<10$ is clearly evident in comparison to the Poisson fluctuations that dominate at $m>15$.

In Section 2, we described the construction of realistic gammaray image simulations. There remain a number of practical steps, which we discuss here. These include removing the coordinate aberration from the Fermi images about a source (Section 4.1), identifying the source center robustly (Section 4.2), and masking source contamination (Section 4.3). We then stack the power spectra from multiple sources (Section 4.5) and determine our final optimized source list (Section 4.6).

\subsection{Converting to Locally Euclidean Coordinates}

At large latitudes, coordinate aberration induces angular structure in the images of even cylindrically symmetric sources. Therefore, we must first approximately flatten the gamma-ray images from Fermi, i.e., transform them to a set of flat coordinates. Because we are interested only in the angular structure of the gamma-ray distribution about the central source $\left(\alpha_{s}, \delta_{s}\right)$, i.e., we are not concerned with its radial structure, fully flattening the images is unnecessary. Rather, it is sufficient to remove the angular distortion.

To do this, we begin with the gamma-ray positions in equatorial coordinates $\left(\alpha_{j}, \delta_{j}\right)$ and perform a rotation to align the reported source position along the polar axis. That is, on the unit sphere, we set the gamma-ray positions to

$$
\begin{aligned}
& x_{j}=\sin \delta_{s} \cos \delta_{j} \cos \left(\alpha_{j}-\alpha_{s}\right)-\cos \delta_{s} \sin \delta_{j} \\
& y_{j}=\cos \delta_{j} \sin \left(\alpha_{j}-\alpha_{s}\right) \\
& z_{j}=\cos \delta_{s} \cos \delta_{j} \cos \left(\alpha_{j}-\alpha_{s}\right)+\sin \delta_{s} \sin \delta_{j},
\end{aligned}
$$

from which we obtain the angular positions by projecting along $\hat{z}$ :

$$
\alpha^{\prime}=\frac{x_{j} \cos ^{-1}\left(z_{j}\right)}{\sqrt{x_{j}^{2}+y_{j}^{2}}} \quad \text { and } \quad \delta^{\prime}=\frac{y_{j} \cos ^{-1}\left(z_{j}\right)}{\sqrt{x_{j}^{2}+y_{j}^{2}}}
$$

\subsection{Maximum-likelihood Center Finding}

The source positions reported by Fermi provide an excellent initial estimate of the source locations. However, these are obtained from a different set of event reconstructions (Pass 7R_V15 SOURCE) from those employed here (Pass 8R2_V6 ULTRACLEANVETO). Moreover, even potentially small offsets provide an obvious systematic uncertainty that will generate spurious power at $m=1$. While we discuss how such an error may be naturally identified in the structure of the angular power spectra directly in Section 4.4.2, we also make an effort to mitigate this directly via an improved estimate of the source location. This also has the effect of treating the mock images in the same fashion as the real data-both will have small offsets in the source location based on the particular photon realization.

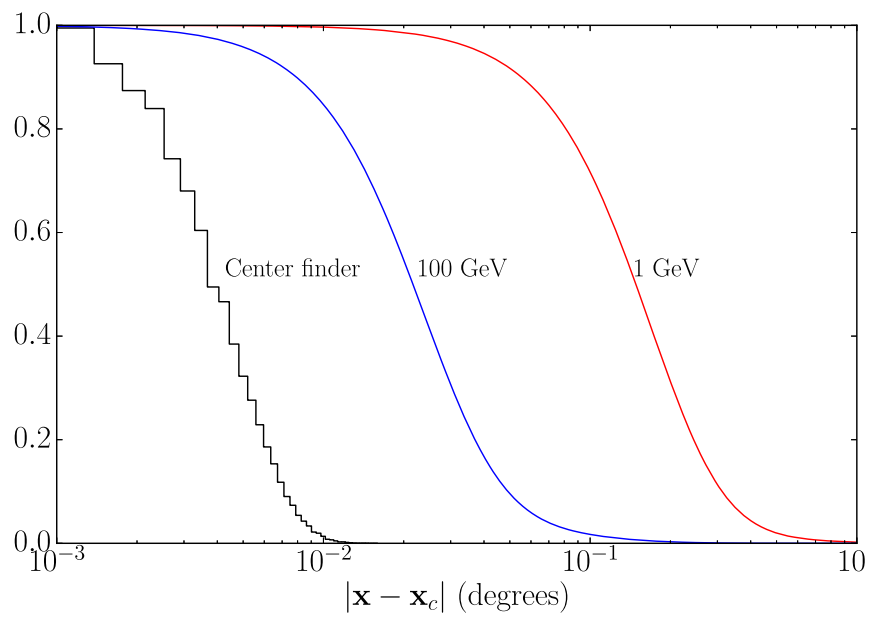

Figure 9. Distribution of displacements between the true and reconstructed centers for a number of mock sources with typical fluences in the $1 \mathrm{GeV}$ $100 \mathrm{GeV}$ band. For reference, the Pass 8R2_V6 ULTRACLEANVETO PSFs for front-converted events at $1 \mathrm{GeV}$ (red) and $100 \mathrm{GeV}$ (blue) are shown. In all cases, the reconstructed center locations are at least an order of magnitude better than the typical PSF width.

To identify the source location, we make a maximumlikelihood estimate of the source location, $\left(\alpha_{s}^{\prime}, \delta^{\prime}{ }_{s}\right)$. The likelihood was taken to be composed of a Gaussian source of known size on top of a uniform background within a specified angular size on the sky. The result is an estimate of the source location and ratio of the source-background fluences. Additional details of the method can be found in Appendix B.

While the Fermi PSF is neither Gaussian nor independent of energy, and thus our likelihood does not formally describe the Fermi response for a point source, both have proven to be adequate approximations for our purpose, producing highly accurate source location estimates. We verified this by generating mock point-source images following the algorithm described in Section 2, which utilizes the fully energydependent Fermi Pass 8R2_V6 PSF, and generating source location estimates. The distribution of offsets is compared to the Fermi Pass 8R2_V6 PSFs at low and high energies in Figure 9. In all cases, our estimate is roughly an order of magnitude better than the characteristic width of the Fermi Pass 8R2_V6, a reflection of the large number of photons in the gamma-ray maps of the bright sources of interest.

We generate a final set of source-centered positions

$$
\alpha_{j}^{\prime \prime}=\alpha_{j}{ }^{\prime}-\alpha_{s}^{\prime} \text { and } \delta_{j}^{\prime \prime}=\delta_{j}{ }^{\prime}-\delta_{s}^{\prime},
$$

which we convert into polar coordinates:

$$
r_{j} \equiv \sqrt{\alpha_{j}{ }^{\prime 2}+\delta_{j}{ }^{\prime 2}} \quad \text { and } \theta_{j}=\tan ^{-1}\left(\delta_{j}{ }^{\prime \prime} / \alpha_{j}{ }^{\prime \prime}\right) \text {. }
$$

It is these $\theta_{j}$ that enter into Equation (16) to construct the object-specific angular power spectra.

\subsection{Source Masking and Contamination Mitigation}

The primary sources of noise in the power spectrum estimates are the photons from the source and in the background. Beyond careful source selection, e.g., removing objects with obvious contaminating neighbors or strong background gradients, there is little that can be done regarding the background. However, this is not true for the source itself. 


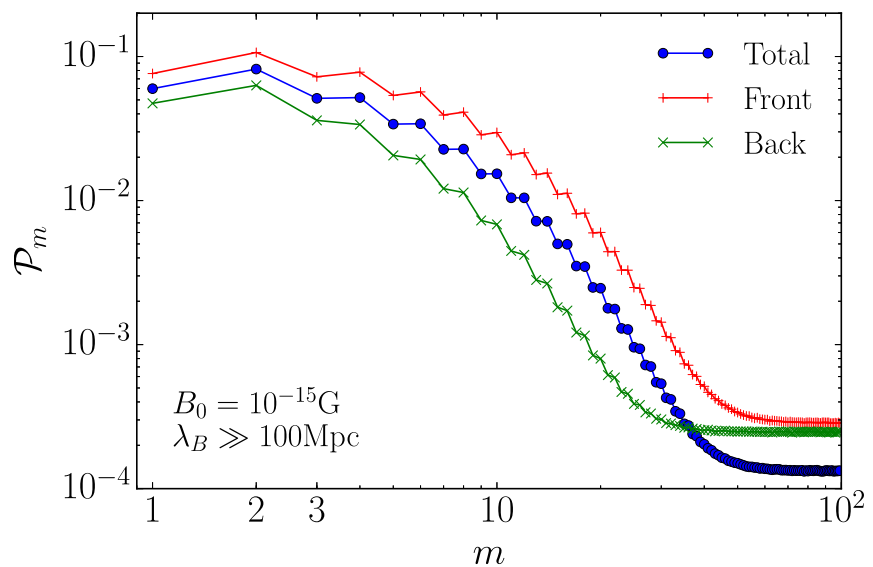

Figure 10. Median mock power spectra for a bright Fermi source containing a large-scale, uniform IGMF constructed from front-converted events (red + ), back-converted events (green $\times$ ), and all events (blue circles). The assumed IGMF strength is $10^{-15} \mathrm{G}$ and $\Gamma_{l}=1.7, z=0.3, \Theta=5^{\circ}, \theta_{\text {jet }}=3^{\circ}, \Gamma_{h}=2.5$, and $E_{p}=1 \mathrm{TeV}$, with on-axis fluence of $5000 \mathrm{ph}$, a background photon density of $50 \mathrm{ph} / \mathrm{deg}^{2}$, and a magnetic field oriented $60^{\circ}$ to the line of sight and $170^{\circ}$ from the horizontal axis. At large $m$, all cases asymptote to the Poisson limit, $\mathcal{P}_{m} \approx N^{-1}$.

Any structure on scales comparable to the PSF width will be erased, eliminating the value of photons near the source. At the same time, the direct photons from a point source contribute dominantly within this region. Therefore, prior to computing the angular power spectrum for each object, we apply an energy-dependent mask, excising the region inside the $68 \%$ containment radius of the Pass 8R2_V6 ULTRACLEANVETO PSF at each energy independently for the front and back detectors. This eliminates at once both a region without a significant anisotropy signal and a substantial source of noise. We assess the impact of variations in the size of the excluded region in Section 5.3, generally finding that it is negligible.

For most objects, the ICC halos extend over angular scales that are large in comparison to the mask, rendering the mask moot. However, for present-day IGMF strengths less than $3 \times 10^{-17}(1+z)^{2} \mathrm{G}$, corresponding to either weak fields or high- $z$, the ICC halo can lie completely within the masked region. ${ }^{12}$ In effect, this simply extends the constraint on ICC halo size already imposed by the finite resolution of the LAT marginally.

\subsection{Example Single-source Power Spectra}

Example average power spectra for a single, bright source are shown in Figures 10-12. These include both the power spectra anticipated from ICC halo models associated with the various IGMF strengths under consideration and those from a variety of potential contaminants. Analytical computations for approximate cases of potential relevance are also collected in Appendix C.

\footnotetext{
${ }^{12}$ This limit arises from combining the angular scale implied by a deflection in Equation (7) and the cosmological evolution of a fixed strength field, given in Equation (15), assuming that the source is sufficiently close that the angular diameter distance is similar to $D_{\mathrm{pp}}$. Thus, we set $\alpha_{\mathrm{def}} / 2 \approx 0.2$, comparable to the typical mask size near $10 \mathrm{GeV}$, from which we obtain $B_{0}=B(1+z)^{-2}=3 \times$ $10^{-17}(1+z)^{2}$. Note that the observed IC gamma-ray energy in Equation (7), $E_{\mathrm{IC}}$, does not redshift.
}

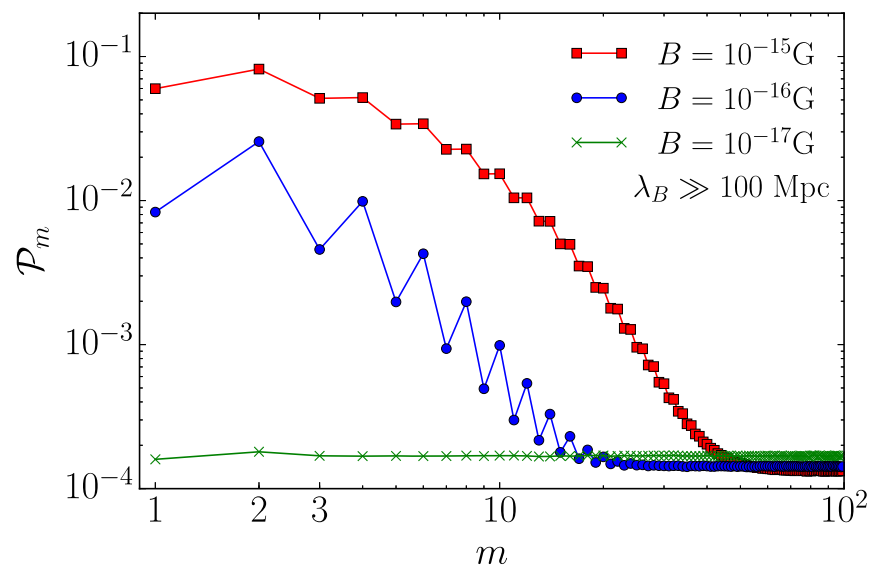

Figure 11. Median mock power spectra for a bright Fermi source assuming different IGMF strengths, $10^{-15} \mathrm{G}$ (red squares), $10^{-16} \mathrm{G}$ (blue circles), $10^{-17} \mathrm{G}$ (green crosses), are shown; decreasing field strength results in a smaller low- $m$ power excess. In all cases, $\Gamma_{l}=1.7, z=0.3, \Theta=5^{\circ}, \theta_{\text {jet }}=3^{\circ}, \Gamma_{h}=2.5$, and $E_{p}=1 \mathrm{TeV}$, with on-axis fluence $5000 \mathrm{ph}$, a background photon density of $50 \mathrm{ph} / \mathrm{deg}^{2}$, and a magnetic field oriented $60^{\circ}$ to the line of sight and $170^{\circ}$ from the horizontal axis.

\subsubsection{ICC Halos}

Power spectra may be made for events converted separately in the front, back, or entirety of the LAT. While frontconverted events have a smaller PSF, and therefore maintain small-scale power more effectively, the improvement in event statistics arising from the near doubling of the gamma-ray number when back-converted events are included produces an overall improvement in the ability to identify ICC halos. This is clearly evident in the comparison between the power at small and large $m$ in Figure 10, which shows each power spectrum class individually.

In all cases at large $m$, the $\mathcal{P}_{m}$ reach the Poisson noise limit, producing a characteristic flattening at $\mathcal{P}_{m} \approx N^{-1}$, indicating the effective number of image photons used. The key discriminant that provides evidence for ICC halos is the disparity between $\mathcal{P}_{2}$ and this floor, for which the power spectrum constructed from all events is largest. Thus, henceforth, we show only the power spectra for the entire event list, i.e., including both front- and back-converted events.

A comparison of the ICC halo signal for different assumptions regarding the IGMF and orientation are shown in Figure 11. In contrast to the null case, the clear signal for an ICC halo is the large quadrupolar power, i.e., $\mathcal{P}_{2}$, in comparison to the Poisson limit. Moreover, the clear oscillatory nature is a signature for the anticipated near bipolar symmetry. Importantly, this "sawtooth" structure is a key systematic diagnostic, differentiating a true ICC halo signal from potential power spectrum contaminants (see the following subsection).

Nevertheless, the magnitude and structure of the power spectrum are strongly sensitive to the assumed IGMF geometry and source viewing angle. For acute viewing angles, i.e., $\Theta \approx \theta_{\text {jet }}$, the large-scale, uniform IGMF models are most significantly distinct from the null case. The power excess extends beyond the even multipoles as a consequence of the breaking of the bipolar symmetry by the intrinsic structure of the jet. When viewed along the jet axis, a near-perfect symmetry exists as the positrons and electrons gyrate in opposite directions, generating halo emission on opposite sides of the source. However, when viewed at angles comparable to the jet width, one component is suppressed by the comparative 

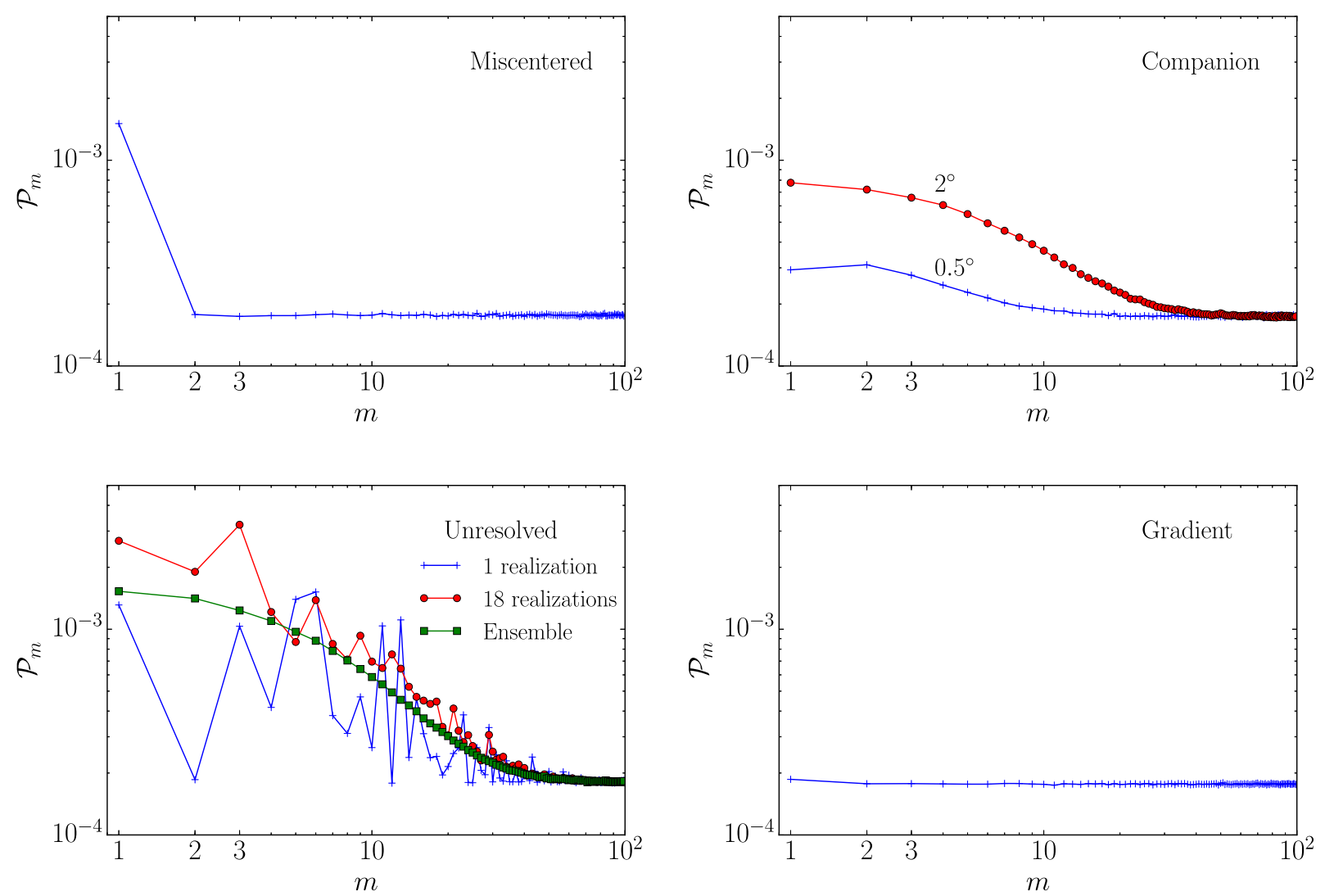

Figure 12. Median mock power spectra for a bright Fermi source without an ICC halo but with various contaminating systematics. Top left: a miscentered image, shifted by 0.05 , corresponding to roughly $10 \%$ of the typical width of the $1 \mathrm{GeV}$ Pass 8R2_V6 ULTRACLEANVETO-front PSF. Top right: a faint companion $\left(150 \mathrm{ph}\right.$ ) that lies well above the Fermi detection threshold (roughly $5 \mathrm{ph}$ ) but below the visual inspection threshold, located at 0.5 (blue) and $2^{\circ}$ (red). Bottom left: a background comprised of many unresolved gamma-ray point sources consistent with the flux distribution of Fermi point sources for a single realization (blue), 18 realizations (red), and an ensemble of realizations (green). Bottom right: a weak gradient in the background comparable to that of the visual inspection threshold. In all cases, $\Gamma_{l}=1.7, z=0.3, \Theta=5^{\circ}, \theta_{\text {jet }}=3^{\circ}, \Gamma_{h}=2.5$, and $E_{p}=1 \mathrm{TeV}$, with on-axis fluence $5000 \mathrm{ph}$ and a background photon density of $50 \mathrm{ph} / \mathrm{deg}^{2}$.

deficit in pair production due to the reduced VHEGR flux. This is insufficient to remove the characteristic "sawtooth" pattern in any case.

Weaker IGMFs produce smaller ICC halo signals, vanishing below $10^{-17} \mathrm{G}$ as a result of the source mask. IGMFs that are much stronger than $10^{-14} \mathrm{G}$ also produce weaker ICC halo signatures in the power spectra as a result of the dilution of the gamma-ray flux due to multiple gyrations. Thus, in principle, apart from a simple detection, which is the focus of this work, it should be possible to characterize a large-scale IGMF given a measurement of the gamma-ray angular power spectra.

Also visible in Figure 11 is a two-zone analogue of a gamma-ray excess at large angular radii from the central source, i.e., the signal described in Figure 7. In the stacked angular power spectrum, this takes the form of a systematic drop in $\mathcal{P}_{m}^{\text {tot }}$ at large $m$ that systematically grows with increasing $B_{0}$. This arises from the ICC halos moving photons from within the central masked region to the outside, where they are included in the angular power spectrum estimate, decreasing the Poisson noise limit. As discussed earlier, exploiting this signal requires knowing the gamma-ray background and radial structure of the PSF to high accuracy a priori.

\subsubsection{Contaminants}

Low-multipole angular power can also be produced by features that are independent of ICC halos. These include systematic errors in the generation of the angular power spectra, angular structure in the Fermi Pass 8R2_V6 ULTRACLEANVETO PSF, and unresolved features in the background. Here, we quantitatively consider each of these. Importantly, none produce the quadrupole-dominated, sawtooth structure in the angular power spectrum characteristic of the bipolar structures associated with the ICC halos.

As has already been described in Section 4.2, the location of the central source may be identified with an accuracy that significantly exceeds the typical PSF width of Fermi. Nevertheless, centering errors combined with the large radial gradients in the gamma-ray flux away from the source center produce a natural source of error in the angular power spectrum (see, e.g., Appendix C.2). To assess the magnitude of this error, we intentionally offset a bright gamma-ray source typical of the Fermi sample by 0.05 , corresponding to roughly $10 \%$ of the typical width of the $1 \mathrm{GeV}$ Pass 8R2_V6 ULTRACLEANVETO-front PSF. As seen in the top-left panel of Figure 12, this leads to spurious power at low multipoles, dominated by the dipole, falling rapidly with $m$ and joining the typical Poisson noise by $m=2$. The large dipole-quadrupole power ratio makes this easily distinguishable from a signal attributable to ICC halos.

Unresolved sources within the background surrounding the primary Fermi AGN also provide a natural source for dipole angular power. This arises in two instances, the first of which is a single neighboring object just below our exclusion threshold. Given that the Fermi detection threshold is 5 ph (Ackermann et al. 2013b), this is unlikely to produce a substantial 
contribution for images comprised of many thousands of events. Adding a source that is obviously visible (150 ph, intermediate to the companions in the left and center panels of Figure 4) produces a notable excess of power at low multipoles (top-right panel of Figure 12). The degree of this excess depends on the location of the contaminating neighbor, becoming larger for more distant unresolved sources. In practice, this is many times over the Fermi detection threshold and would already be excluded. Much weaker companions would produce a correspondingly weaker contribution to the power spectrum. In all cases, as before, it is dominated by the dipole component and fails to exhibit the sawtooth morphology.

The second way in which unresolved sources enter is through the origin of the background itself. At high energies $(>50 \mathrm{GeV})$, nearly the entirety of the extragalactic gamma-ray background has been resolved into point sources (Ackermann et al. 2016b). Extending this to lower energies results in a clustering of photons about the brightest background objects and therefore low-multipole power arising from a handful of unresolved neighbors. ${ }^{13}$ In practice, the extended fields about individual targets in Table 1 do include known 3FGL sources with up to $40 \mathrm{ph}$, well above the Fermi detection threshold. Thus, shown in the bottom-left panel of Figure 12 is the angular power spectrum arising from a background comprised of a population of sources below the $40 \mathrm{ph}$ threshold and distributed according to the 2FGL flux distribution (Abdo et al. 2010a; Broderick et al. 2014b). Because the latter is formally divergent, we filled in the background from the highflux end, beginning just below the Fermi threshold and stopping when the required number of background photons was obtained. For all of the background sources, we adopted a photon spectral index of 2.4 , consistent with the $1 \mathrm{GeV}-$ $100 \mathrm{GeV}$ background (Abdo et al. 2010b; Ackermann et al. 2015a).

Here, it is useful to distinguish between random realizations of the background sources, i.e., the positions and fluxes of the unresolved sources that comprise the background, and random realizations of photons drawn from the sources. Even after averaging over a full ensemble of photon realizations, a single background source realization produces a stochastic angular power spectrum exhibiting large variations at low multipoles, shown by the blue line in the bottom-left panel of Figure 12. This is a result of the structure imposed on the background via the locations and strengths of the background sources and cannot be overcome by collecting additional observations. Averaging over realizations of the background sources and photons results in a smooth angular power spectrum, similar to that arising from a nearby, unresolved source (green line in the bottom-left panel of Figure 12).

In principle, we can directly measure this background angular power spectrum using nearby empty fields. However, our ability to remove it is fundamentally limited by the variance due to the moderate number of background realizations presented by the source list in Table 1 . Nonetheless, even after averaging over 18 sources (the number of sources in Table 1), the fluctuations are already substantially reduced (red line in bottom-left panel of Figure 12). Regardless, the

\footnotetext{
${ }^{13}$ This is extremely pessimistic. Even at high latitudes, the smooth, Galactic contribution to the gamma-ray background is substantial. Nevertheless, this gives an extreme estimate of the impact of a highly structured background.
}

stochastic structure lacks the telltale sawtooth morphology of the ICC halos.

The azimuthal structure of the Fermi Pass 8R2_V6 ULTRACLEANVETO PSF will also generate angular power within the source photons. Note that this is not true for the distribution of the uniform gamma-ray background-even a highly anisotropic PSF cannot impart structure to a uniform field of photons. The angular structure of the Fermi Pass 8R2_V6 ULTRACLEANVETO PSF arises from the square geometry of the LAT (see also Section 2.1). The instantaneous PSF exhibits only 5\% variations in the PSF at the energies of interest (Ackermann et al. 2012a). Over timescales short in comparison to years, this is substantially reduced by the rotation of Fermi during solar tracking and the eightfold symmetry of the LAT.

In Appendix D, we make an estimate of the residual PSFinduced angular power, assuming that the cumulative gammaray image is comprised of many epochs during which the roll angle of Fermi is highly correlated. The duration of these epochs correspond to roughly the time for Fermi to rotate by $45^{\circ}$, after which it effectively rotates through the entirety of the PSF as a result of the LAT's square geometry. Presuming that the fluence is relatively evenly distributed over the past eight years, even for optimistic assumptions regarding the structure of the PSF, the estimated residual angular power is less than $1 \%$ of the anticipated Poisson noise. Moreover, it vanishes identically for $m=2$ as a result of the LAT's symmetry, exhibiting power only for $m=4$ and its harmonics.

Finally, we considered a linear gradient in the background photon density. Because we explicitly select sources for which there is no apparent large-scale gradients in the background, we again set the value to our effective detection threshold, corresponding to a variation in the photon density of $20 \%$ across the $4^{\circ}$ image. As shown in the bottom-right panel of Figure 12, the impact on the angular power spectrum is very small, weakly modifying the dipole power primarily.

It is important to note that there are two key features regarding all of the potential systematic uncertainties arising from contaminants. First, their shape is qualitatively different from the distinct signatures of the bimodal ICC halos. Second, their magnitude is far smaller than that expected from the ICC halos arising from a large-scale, uniform IGMF. As a result, they should be readily distinguishable.

\subsection{Combining Multiple Sources}

Finally, to increase the fidelity of the angular power spectrum, we stack the estimates from multiple sources. Unlike stacking the images directly, this preserves the anisotropic signal; a rotation of any image, corresponding to setting $\theta_{j} \rightarrow \theta_{j}+\varphi$, leaves $\mathcal{P}_{m}$ unchanged, as may be verified by inspection of Equation (16). It does, however, reduce the intrinsic scatter in the power spectrum estimate.

In principle, this may be optimized via the weighting assigned to individual sources-images with higher numbers of intrinsic photons will produce better intrinsic power spectrum estimates and thus may be given additional weight in the stacking processes. The natural way to do this is through variance weighting, giving the smallest variance in $\mathcal{P}_{m}$ at each $m$. In practice, we found that the dominance of the source counts by a handful of objects (e.g., Mkn 421) led to an associated dominance of the power spectra estimate, eliminating much of the power of the stacking process. 
It is also possible to exploit the spectra of the ICC halos and/ or the redshift dependence of the ICC halo extent to provide more optimal weightings. We found that no such effort made a substantial impact on the ability to distinguish the power in the quadrupole and the neighboring odd multipoles, the key observable for our bimodal ICC halos. The reason is simply that while the ICC halos are typically harder than the gammaray background, they are only marginally so, limiting the ability to spectrally separate the two components.

Therefore, we define our stacked power spectrum by the arithmetic average of the individual source power spectra,

$$
\mathcal{P}_{m}^{\text {tot }}=\frac{1}{N_{\text {src }}} \sum_{\text {src }} \mathcal{P}_{m}^{\mathrm{src}},
$$

where $\mathcal{P}_{m}^{\text {src }}$ is the single-source power spectrum defined by Equation (16).

\subsection{Source List Optimization}

The ability to generate simulated realizations of the Fermi sky enables us to theoretically optimize the list of Fermi sources that are ultimately stacked. That is, apart from gross properties of the sources (e.g., SEDs and redshifts), we can select the group most likely to collectively produce the apparent signatures of ICC halos in the stacked angular power spectrum without looking at the actual structures of these images.

The SEDs described in Section 3.1 provide guidance on which sources are likely to be bright above a TeV. However, while this is a necessary condition, it is not sufficient to produce bright ICC halos. The halo itself is impacted by the source distance (among other parameters that are marginalized over). The ability to detect the halo is impacted by the local background. Thus, armed with the ability to simulate halos from the 27 sources that are sufficiently isolated, appear in 2FHL and 3LAC, and have known redshifts, we apply a final optimization step designed to maximize the ability to detect ICC halos.

We begin by defining a halo-model specific detection likelihood statistic,

$$
P_{\mathrm{det}}=\int d p P_{>\mathcal{P}_{2}}^{\mathrm{h}}(p) P_{\mathcal{P}_{2}}^{\mathrm{n}}(p)
$$

where $P_{\mathcal{P}_{2}}^{\mathrm{n}}(p)$ is the probability of finding $\mathcal{P}_{2}^{\text {tot }}=p$ in the null case, i.e., without a halo, and $P_{>\mathcal{P}_{2}}^{\mathrm{h}}(p)$ is the cumulative probability associated with $\mathcal{P}_{2}^{\text {tot }} \geqslant p$ when a halo is present. This is the probability that the $m=2$ power from the given halo model exceeds that from the null case, marginalized over the probabilities of both. For the null case, $P_{\text {det }}=1 / 2$, i.e., in the absence of an ICC halo, the probability that $\mathcal{P}_{2}$ will exceed that from the null case is simply $50 \%$. This translates directly into the probability that a given halo model will produce excess power in the quadrupole, i.e., the probability that an ICC halo is detectable. From a collection of $N_{\text {real }}$ realizations of the full sample of isolated, hard Fermi AGNs, we estimate $P_{\text {det }}$ via

$$
P_{\mathrm{det}} \approx \frac{1}{N_{\text {real }}} \sum_{\text {real }} \frac{1}{51} \sum_{m=50}^{100} \Theta\left(\mathcal{P}_{2}^{\text {tot }}-\mathcal{P}_{m}^{\text {tot }}\right),
$$

where $\Theta(x)$ is the Heaviside function and we have used the high- $m$ power spectra as a proxy for the null case. This is necessarily a function of the particular set of sources included, changing as a result of all of the systematic inputs into the construction of the ICC halo models and the stacked power spectra. Thus, for a given halo model, we can optimize the list of input sources by maximizing the simulated values of $P_{\text {det }}$.

This maximization is achieved in a restricted sense in practice: we order the list of sources by intrinsic $\mathrm{TeV}$ brightness and then construct a sequence of source lists, each comprised of the preceding list and the next-brightest $\mathrm{TeV}$ source. For example, the first list includes Mkn 421, and the second, Mkn 421 and 3FGL J2347.0+5142, etc. The value of each source is then evaluated when it is first included; if it increases $P_{\text {det }}$ it is kept, if it decreases $P_{\text {det }}$ it is removed. This procedure results in a set of optimal sources for each halo model.

This list varies between halo models due to the differences in the ICC halos produced. As a result in Table 1, we present four separate samples corresponding to four different choices of $B_{0}$ for the uniform IGMF halo models: $B_{0}=10^{-17} \mathrm{G}, 10^{-16} \mathrm{G}$, $10^{-15} \mathrm{G}$, and $10^{-14} \mathrm{G}$. In practice, they are nested: the first sample is comprised of the brightest four sources, the second sample includes the first and the next six sources, etc. This is a result of the smaller apparent sizes of the halos associated with weaker IGMFs, and thus preferring a brighter and nearer AGN sample. It is this collection of source samples that we will use henceforth.

It is important to note that this optimization procedure was performed entirely using the simulated images. That is, apart from the source SEDs and measured redshifts, the actual source structure played no role. Hence, we have in no way begged the question by having done so. Rather, this is precisely the chief advantage of simulating the data-it permits identifying the key signatures of the ICC halos and optimizing the procedure to detect them.

\section{Monte Carlo Confidence Level Estimates}

The median angular power spectra presented in the previous section, while indicative of the impact of different image features, are poor representations of the angular power spectra associated with a single source realization. The fractional uncertainty in the power at a given multipole for a single image is of order unity (see Appendix C.3), implying large deviations are typical. This is ameliorated by stacking the power spectra from multiple images as described in Section 4.5, which formally decreases the scatter by roughly $N_{\mathrm{src}}^{-1 / 2}$. However, variations in the underlying intrinsic source and IGMF properties act to increase the fluctuations in $\mathcal{P}_{m}$.

Here, we report the resulting 95\% confidence level regions for the various ICC halo models, and thereby IGMF models. The meaning of these is similar to that of a likelihood; they represent the probability of finding a given value of $\mathcal{P}_{m}^{\text {tot }}$ at a specified $m$ for a given IGMF. As such, they present a natural way to assess the single realization afforded by the actual Fermi data.

Given the approximately bimodal nature of the ICC halos, we focused on two primary observables: the quadrupolar power $\mathcal{P}_{2}^{\text {tot }}$ and the sawtooth morphology. The latter is primarily in the service of separating the contributions to the angular power spectrum from the ICC halos from other sources, including the potential sources of systematic error discussed in Section 4.4.2.

There are a number of additional potential observables. Typically, strong correlations between nearby multipoles limit the ability to leverage deviations for many $m$ to improve 
statistical significance. Nevertheless, key systematics may be assessed using the distribution of $\mathcal{P}_{m}^{\text {tot }}$ at large $m$, set by the Poisson noise and, therefore, the effective number of gammarays used to construct the power spectrum estimate.

Thus, the essential experiment is to compare the low- $m \mathcal{P}_{m}^{\text {tot }}$ to the predictions of the various IGMF/halo models. Confidence in the simulation of the Fermi sources and their subsequent stacked angular power spectrum is obtained by comparing their large- $m$ characteristics. The confidence with which any IGMF may be detected or excluded is, then, set by the degree to which the measured $\mathcal{P}_{m}^{\text {tot }}$ is inconsistent with the predicted value at, for example, $m=2$.

The key theoretical input is the anticipated distribution of $\mathcal{P}_{m}^{\text {tot }}$ for a given IGMF model. Here we describe how we estimate the ranges over which the angular power spectra can vary for a given halo model and what these ranges are for the particular IGMF models described in Section 2.

\subsection{Generating Mock Fermi Samples}

The confidence with which a given IGMF can be detected or excluded based upon the gamma-ray image angular power spectra depends critically on a quantitative estimate of the range of, distribution of, and correlations among the $\mathcal{P}_{m}$ for the stacked power spectra of the Fermi sample. To assess this, we perform a Monte Carlo sampling of the Fermi sample and its associated $\mathcal{P}_{m}^{\text {tot }}$. This synthesizes the steps described in Sections 2-4 for a large-scale IGMF of various strengths.

In summary, for each realization of the Fermi sample, we generate a set of realizations of each source in Table 1 consistent with their known intrinsic properties (e.g., number of source photons, $N_{\mathrm{ph}}$, and surface density of background photons, $\mathcal{B}$ ) and our estimates for the distributions of their unknown intrinsic properties (e.g., $\Gamma_{h}, \theta_{\text {jet }}, \Theta$, etc.). Within each image, we choose the number of source and background photons from a Poisson distribution, taking $N_{\mathrm{ph}}$ and $\pi R_{\mathrm{img}}^{2} \mathcal{B}$ as the means of the distributions, respectively (see Table 1). The source photons are distributed between the direct component and the ICC halo according to the ICC halo model as described in Section 2.2, with the local strength and orientation of the IGMF permitted to vary as described in Section 3.4.

The resulting list of realized $\mathcal{P}_{m}^{\text {tot }}$ provides a proxy for the distribution of stacked angular power spectra associated with a given IGMF model. To quantify this distribution, we quote one-sided 95\% confidence level regions, i.e., the values of $\mathcal{P}_{m}^{\text {tot }}$ that $95 \%$ of the realizations lie above, and independently the value that $95 \%$ lie below; because they are one-sided, these are subtly different from normal $2 \sigma$ errors. This procedure of using one-sided confidence levels enables rejecting a model hypothesis at $95 \%$ confidence if it lies outside the one-sided halfinterval. We also use the sample to explore the correlations between the stacked angular power at different multipoles; when ICC halos contribute substantially to the $\mathcal{P}_{m}^{\text {tot }}$, the multipoles are generally strongly correlated, maintaining the sawtooth structure.

\subsection{Anticipated Inverse Compton Halo Power Spectra}

We now turn to the probability distributions of $\mathcal{P}_{m}^{\text {tot }}$ for the various halo/IGMF models of interest. Note that in many cases the power at nearby multipoles are strongly correlated.

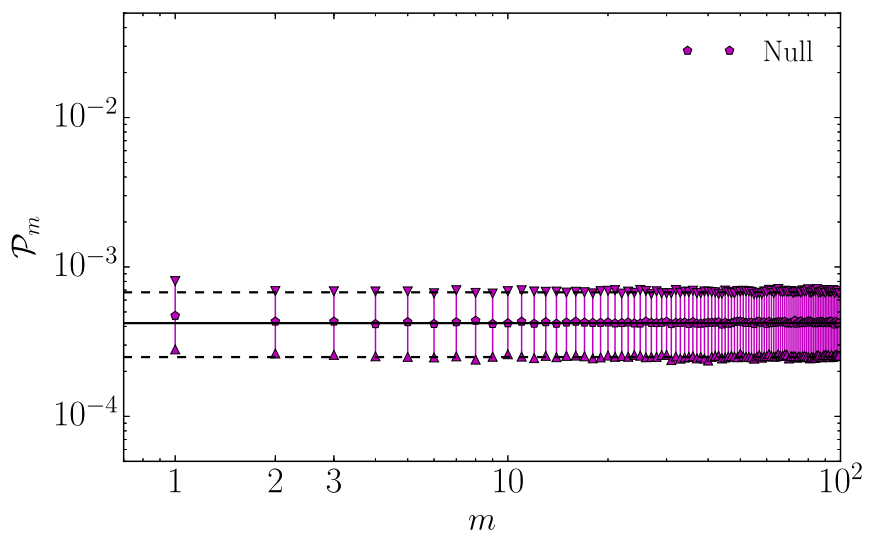

Figure 13. Mock angular power spectrum distributions when no ICC halos are present. The median value is shown by the filled pentagon; the triangles denote the one-sided 95\% confidence interval. In comparison, the black solid and dashed lines show the Poisson noise limit and its one-sided 95\% confidence intervals, respectively, defined collectively by combining the distributions of all multipoles. This is produced with the $10^{-15} \mathrm{G}$, large-scale, uniform IGMF optimized source list.

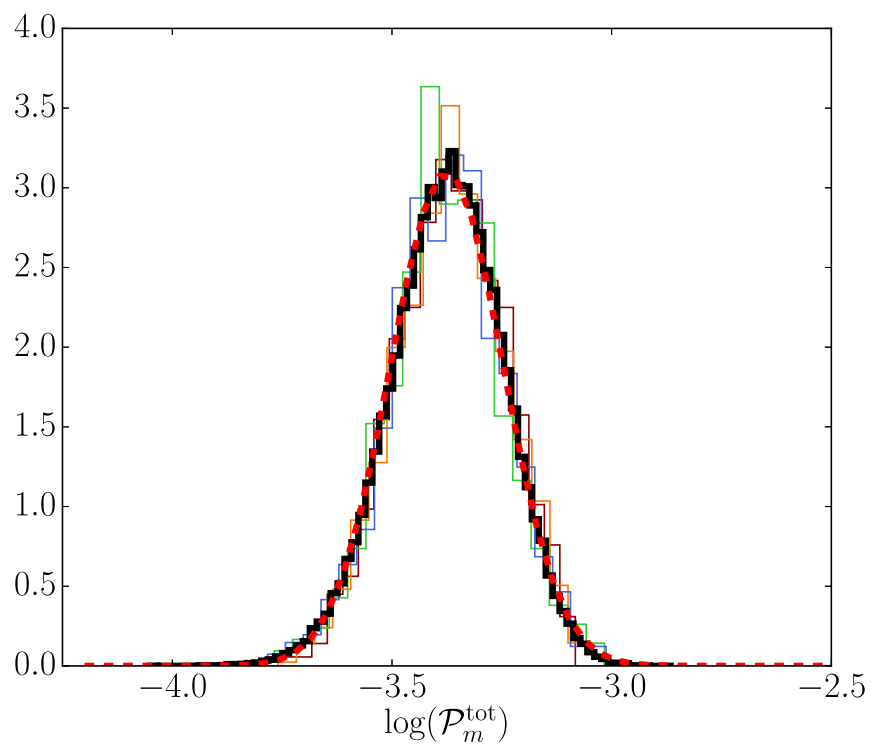

Figure 14. Distribution of the mock $\mathcal{P}_{m}^{\text {tot }}$ about the median for the first four multipoles ( $m=2,3,4,5$ from red to blue) and the first 100 multipoles combined (black). For comparison, a log-normal fit is shown (red dashed line) with mean -3.38 and standard deviation 0.129 . This is produced with the $10^{-15} \mathrm{G}$, large-scale, uniform IGMF optimized source list.

\subsubsection{Null Case}

We begin with the null hypothesis - the absence of any ICC halo component. In this case, the image consists solely of the central point source and uniform background for each object in the Fermi sample. This represents the baseline against which all subsequent models that include ICC halos are compared. Here, we use the $10^{-15} \mathrm{G}$, large-scale, uniform IGMF optimized source list.

The median angular power spectrum, including front- and back-converted events, and its $95 \%$ one-sided confidence limits are shown in Figure 13. As anticipated, there is no structure in this case-the power spectrum is well-fit by a constant corresponding to the effective Poisson noise limit. The distributions about the median values of $\mathcal{P}_{m}^{\text {tot }}$ are uncorrelated between multipoles and well-fit by a log-normal distribution, shown in Figure 14. 

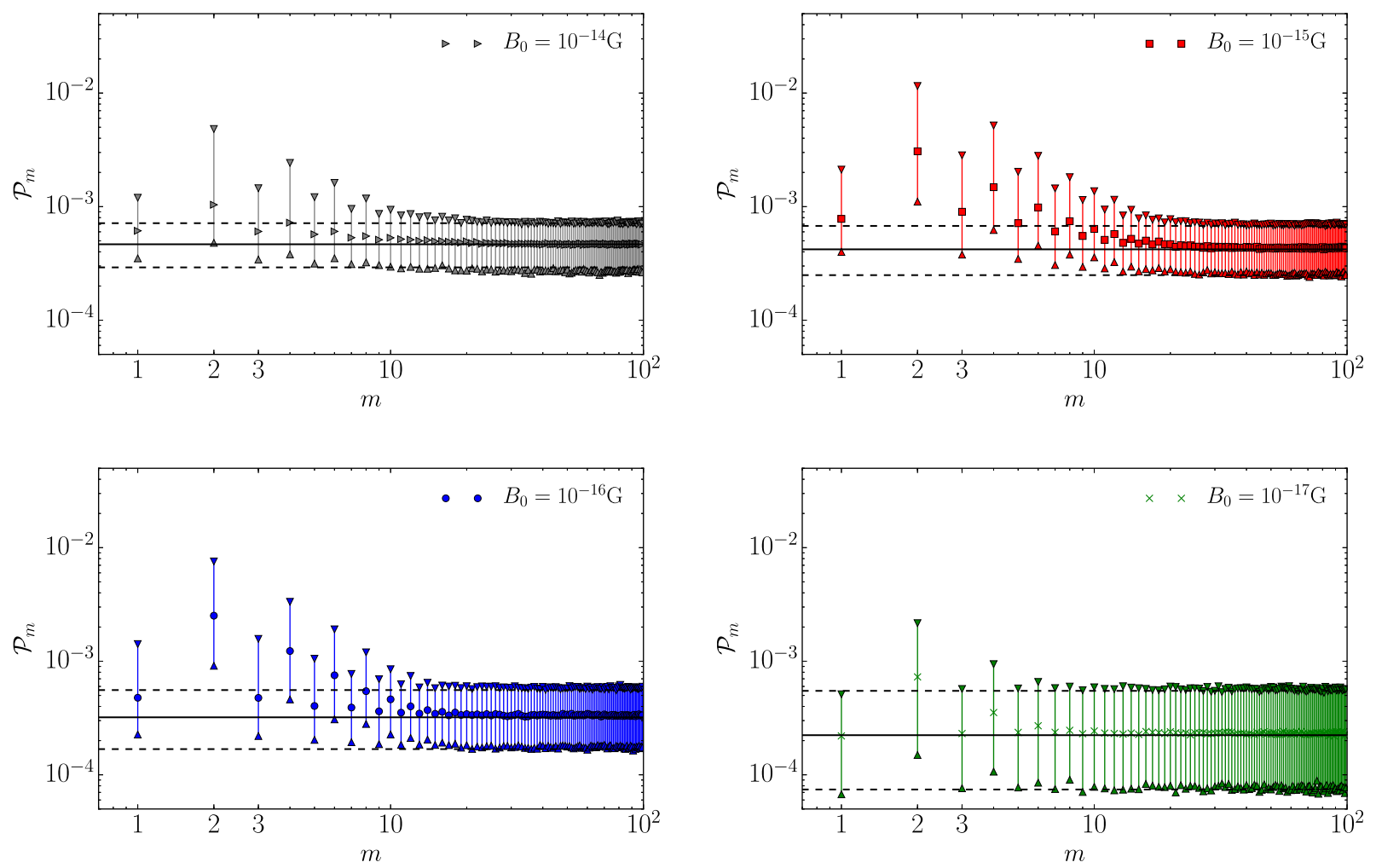

Figure 15. Mock angular power spectra distributions when ICC halos associated with a large-scale, uniform IGMF are present for $B_{0}=10^{-15} \mathrm{G}$ (gray triangle, upper left), $B_{0}=10^{-15} \mathrm{G}$ (red squares, upper right), $10^{-16} \mathrm{G}$ (blue circles, lower left), and $10^{-17} \mathrm{G}$ (green crosses, lower right). The median value is shown by the filled points; the triangles denote the one-sided $95 \%$ confidence interval. In comparison, the black solid and dashed lines show the Poisson noise limit and its one-sided $95 \%$ confidence intervals, respectively, defined collectively by combining the distributions of multipoles with $m \geqslant 50$. Each case was produced using the associated optimized source list (see Table 1).

We would expect any contaminating systematic (e.g., centering errors or dim neighbors) to contribute in a fashion similar to that seen for the single-source power spectra presented in Section 4.4.2.

\subsubsection{Large-scale, Uniform IGMF}

We show the distributions of stacked angular power for a variety of magnetic field strengths in Figure 15. These all exhibit excess power at low $m$, becoming less significant as the field strength nears $10^{-17} \mathrm{G}$, as anticipated from the singleimage power spectra in Section 4. For $B_{0}>10^{-17} \mathrm{G}$, the deviations from Poisson noise are substantial, increasing with increasing field strength; for $B_{0}=10^{-15} \mathrm{G}$, the excess power extends to $m \approx 30$. Importantly, note that for $B_{0}=10^{-15} \mathrm{G}$ and $10^{-16} \mathrm{G}$ the quadrupolar power is inconsistent with that for the null hypothesis, implying that the angular power spectrum will immediately distinguish between these two IGMF/halo models.

The breaking of the bipolar symmetry is clearly apparent in the stacked angular power, corresponding to large power at odd multipoles. This is a natural consequence of the larger probability of the viewing angles near the maximum permitted (see Section 2). Nevertheless, the sawtooth structure is directly evident in the medians of the $\mathcal{P}_{m}^{\text {tot }}$ for all cases.

Less obvious is that this remains true in individual realizations despite the large apparent variations implied by the extent of the 95\% confidence regions; as seen in Figure 16, the power in all multipoles are strongly correlated, moving the

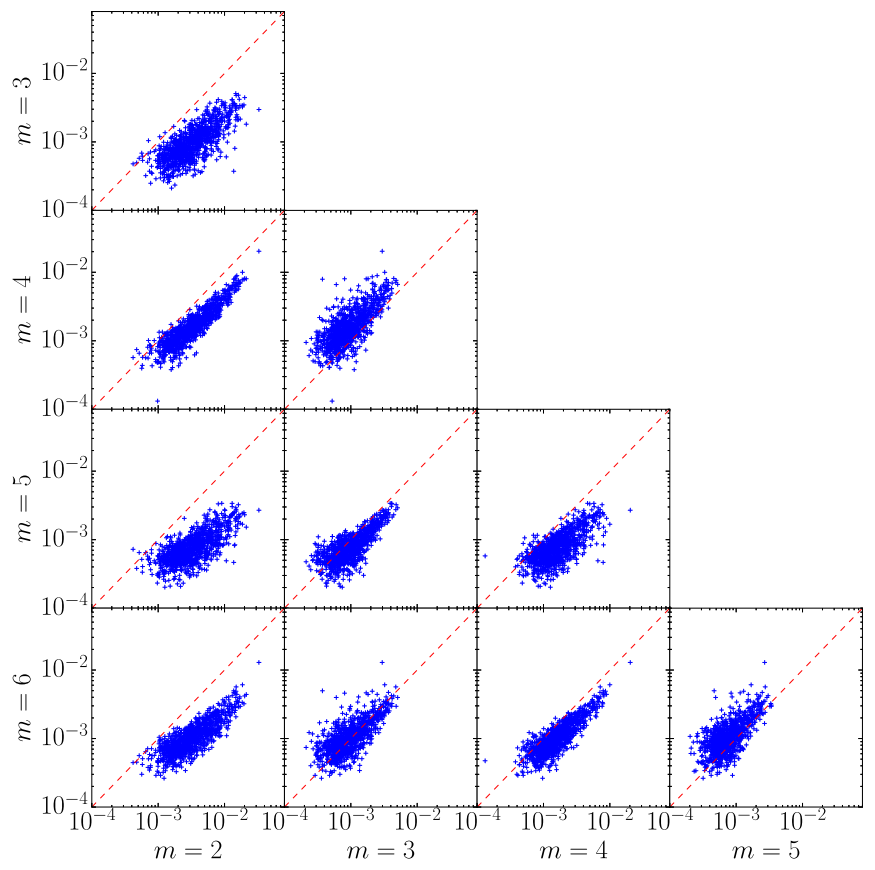

Figure 16. Pairwise correlations between the angular power in the $m=2,3,4$, and 5 multipoles for each mock realization of the Fermi sample when ICC halos are associated with a large-scale, uniform IGMF with $B_{0}=10^{-15} \mathrm{G}$. For reference, the one-to-one line is shown by the red dashed line. This is produced with the same set of realizations used to construct the associated panel of Figure 15. 


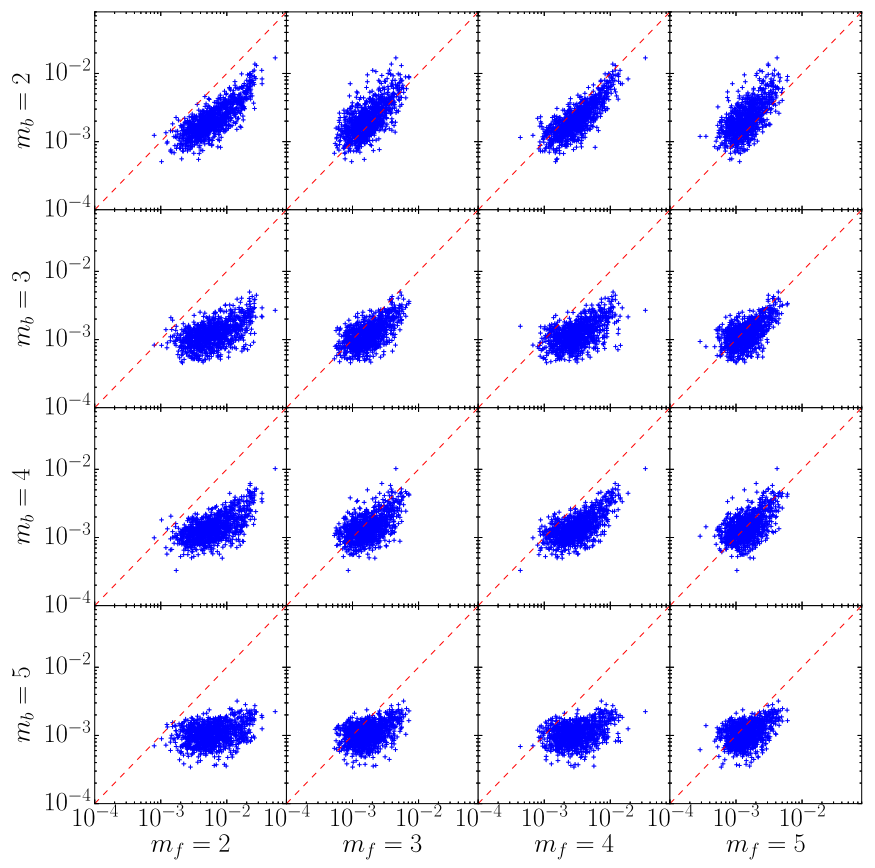

Figure 17. Pairwise cross-correlations between the angular power in the $m=2,3,4$, and 5 multipoles obtained from the front- $\left(m_{f}\right)$ and back-converted $\left(m_{b}\right)$ events for each mock realization of the Fermi sample when ICC halos are associated with a large-scale, uniform IGMF with $B_{0}=10^{-15} \mathrm{G}$. For reference, the one-to-one line is shown by the red dashed line. This is produced with the same set of realizations used to construct the associated panel of Figure 15.

sawtooth pattern up and down together. This is particularly true for the quadrupole, which overwhelmingly dominates all of the odd multipoles in more than $99 \%$ of the realizations, failing to do so only in the most pessimistic cases. This remains true for the front- and back-converted events independently, as shown in Figure 17, which shows the cross-correlation of the two event populations. Thus, it is always possible to distinguish the large-scale, uniform IGMF model from the many potential sources of contamination in the angular power spectrum that show a smoothly falling excess of power at low-m generally. The strong correlation among multipoles also means, however, that it remains possible that the power excess is low at all $m$, providing a fundamental limit on the minimum $B_{0}$ that can be reliably differentiated from the null case.

\subsection{Sensitivity to Source-Halo Confusion}

How the potential confusion among the source, halo, and background photons is dealt with modifies the number of halo photons at large angular separations, and thus potentially the significance with which ICC halo contribution to the power spectrum may be identified. We therefore consider modifications to the scheme described in Section 2.2, extending the "source" region, i.e., the region within which photons will be assumed to contribute to the number of source photons, to the 95\% containment radius of the Pass 8R2_V6 ULTRACLEANVETO PSF. As discussed in Section 2.2, this extends to large angular radii, typically well beyond the point at which an extended component would be clearly visible. Nevertheless, as shown in Figure 18, this has little impact on the ability to identify ICC halos in the angular power spectrum.

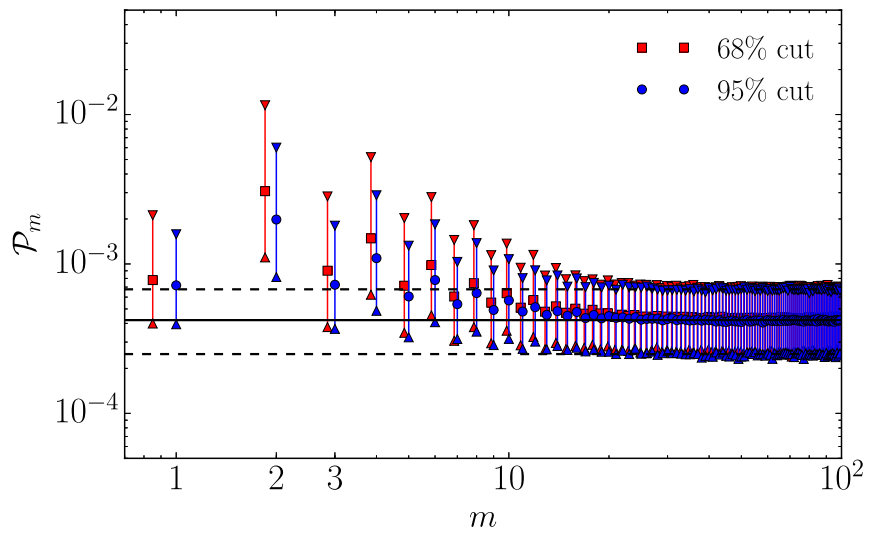

Figure 18. Mock angular power spectrum assuming a large-scale, uniform IGMF with $B_{0}=10^{-15} \mathrm{G}$ when the "source" region is $68 \%$ and $95 \%$ of the Pass 8R2_V6 ULTRACLEANVETO PSF, respectively. The median value is shown by the filled points; the triangles denote the one-sided $95 \%$ confidence intervals. In comparison, the black solid and dashed lines show the Poisson noise limit and its one-sided $95 \%$ confidence intervals, respectively, defined collectively by combining the distributions of multipoles with $m \geqslant 50$. This is produced with the $10^{-15} \mathrm{G}$, large-scale, uniform IGMF optimized source list.

\subsection{Future Prospects with Fermi}

The significance estimates shown in Figures 13 and 15 are all for the current Fermi data set. However, the operational lifetime of Fermi may be considerably longer. Therefore, we also consider a gamma-ray data set that is doubled in size, i.e., the results of 16 years of Fermi observations. To facilitate a direct comparison and in the interest of simplicity, we keep the source lists identical.

As shown in Figure 19, the significance with which a largescale, uniform IGMF can be detected or excluded is significantly improved. Of $10^{3}$ realizations that assume an IGMF strength of $B_{0}=10^{-15} \mathrm{G}$, none were found that had a $P_{2}$ below the $95 \%$ confidence upper limit of the null model. Thus, it would be possible to detect or exclude ICC halos associated with moderate IGMF strengths with significances well above $3 \sigma$.

More importantly, the multipoles for which this may be done extend beyond the quadrupole and includes the $m=4$ mode. At $B_{0}=10^{-15} \mathrm{G}$, this may be similarly excluded or detected with significance $\gtrsim 3 \sigma$; of $10^{3}$ realizations, four lie below the $95 \%$ confidence upper limit of the null model. As a result, should any of the systematic contaminants described in Section 4.4 .2 be present (e.g., weak neighbors, unresolved backgrounds, etc.), the additional statistical power afforded by the increased fluence may prove critical to verifying any putative halo signal. However, the range of IGMF strengths that may be detected or excluded is not improved substantially.

Note that this provides only a very pessimistic view of the value of an extended Fermi mission, even within the narrow confines of the detection of anisotropic ICC halos. A doubling of gamma-ray fluence not only increases the number of photons available in the sources listed in Table 1 but improves the significance with which structure can be identified in sources not listed in Table 1. As a result, the collection of Fermi sources that contribute positively to $P_{\text {det }}$ will grow beyond those in Table 1 at all values of $B_{0}$. As a result, the ability to detect ICC halos will benefit from an increased collection of sources, which we have not addressed, as well as the improved fluence. 

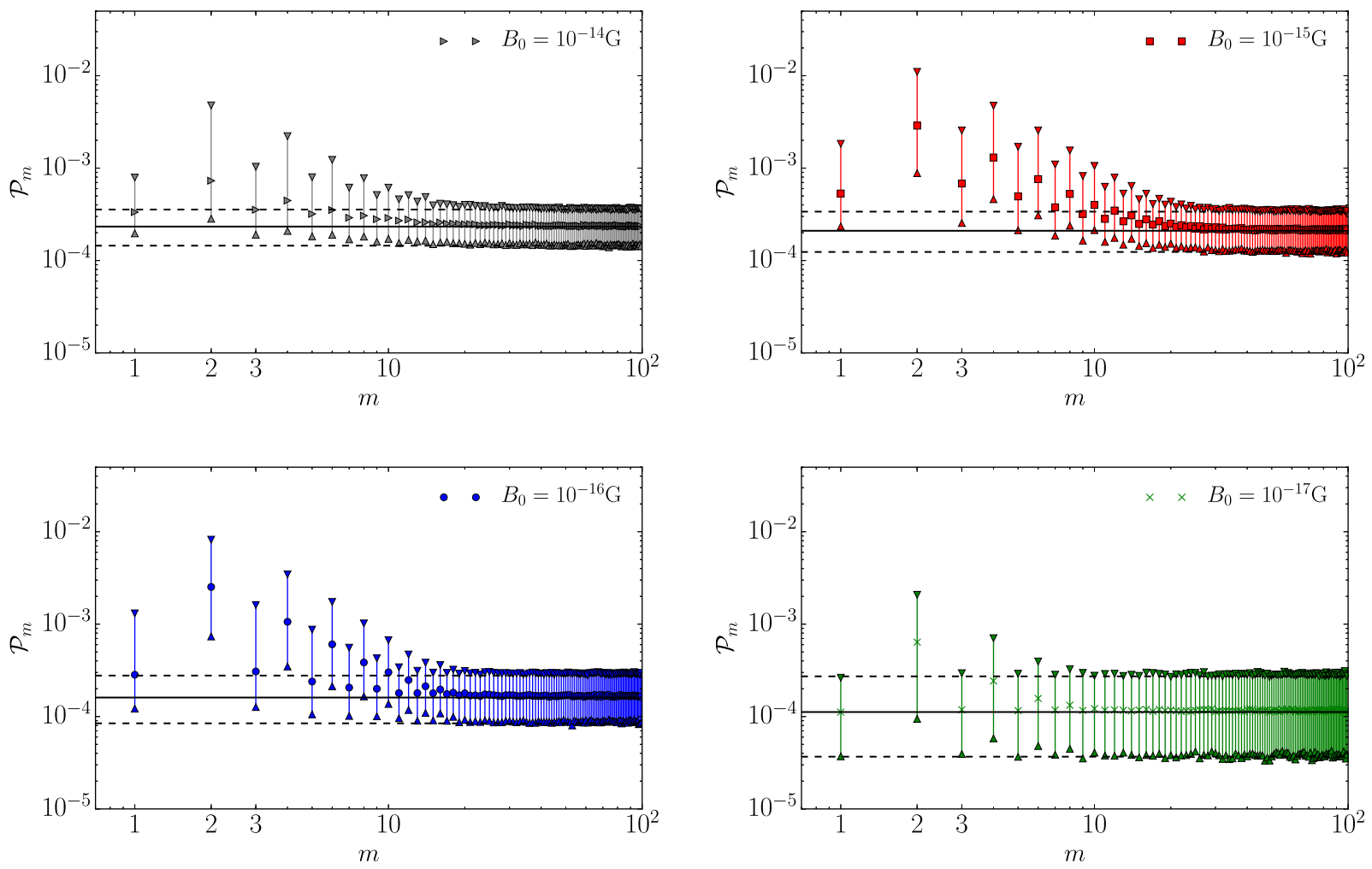

Figure 19. Mock angular power spectra distributions with double the number of photons (i.e., a projected total of 16 years of Fermi observations), when ICC halos associated with a large-scale, uniform IGMF are present for $B_{0}=10^{-15} \mathrm{G}$ (gray triangle, upper left), $B_{0}=10^{-15} \mathrm{G}$ (red squares, upper right), $10^{-16} \mathrm{G}$ (blue circles, lower left), and $10^{-17} \mathrm{G}$ (green crosses, lower right). The median value is shown by the filled points; the triangles denote the one-sided $95 \%$ confidence interval. In comparison, the black solid and dashed lines show the Poisson noise limit and its one-sided $95 \%$ confidence intervals, respectively, defined collectively by combining the distributions of multipoles with $m \geqslant 50$. Each case was produced using the associated optimized source list (see Table 1).

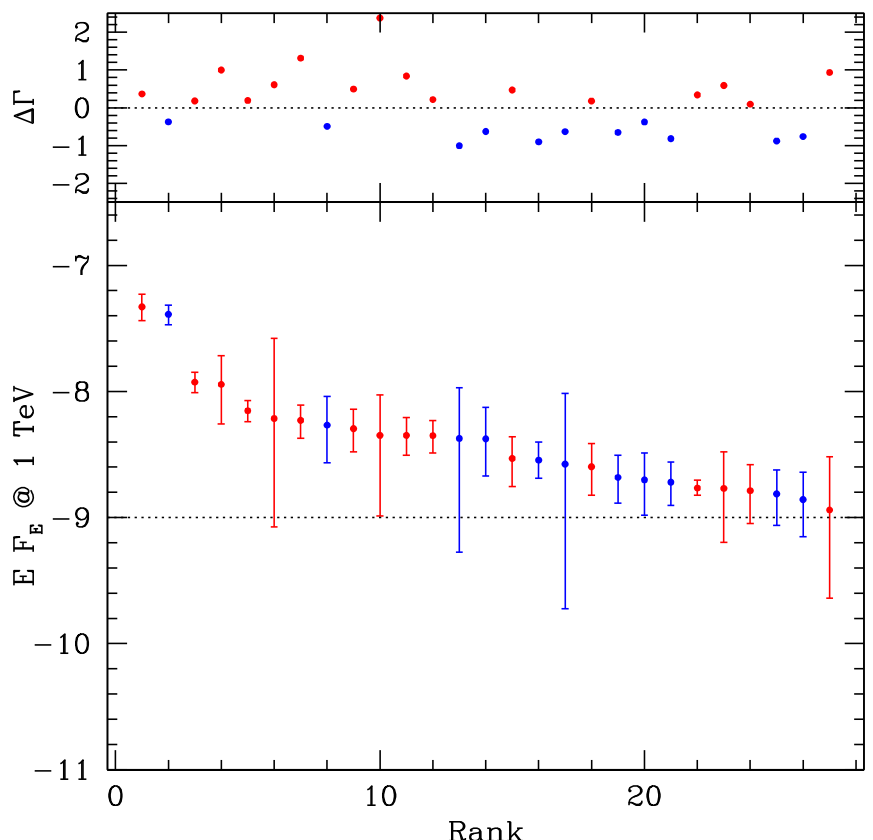

Figure 20. Rank-ordered list of TeV fluxes. The nominal cutoff is shown by the dotted line. Sources are ranked by the $1 \sigma$ lower limit on the TeV fluxes. Sources are color coded by SED type: convex $\left(\Gamma_{h}>\Gamma_{l}\right)$ and concave $\left(\Gamma_{h}<\Gamma_{l}\right)$ SEDs are shown in red and blue, respectively.

\section{Conclusions}

In Broderick et al. (2016), we presented a semi-analytical computation of ICC halos and the generation of realizations around Fermi sources. There we found that the putative ICC halos that surround bright VHEGR sources are generally anisotropic. The origin of the anisotropy depends primarily on the structure of the IGMF in the vicinity of AGNs, and otherwise weakly on its remaining intrinsic and observational properties. For small-scale, tangled IGMFs, the halo structure is driven by the beamed nature of the VHEGR emission; for large-scale, uniform IGMFs, the halo structure is a result of the opposite gyration of electrons and positrons coupled with the geometry of IC scattering in the high-energy limit.

In all cases, however, the structure of the gamma-ray map is bimodal. Here, we suggest an explicit experiment to detect ICC halos using this bimodal structure that is insensitive to the many systematic uncertainties in the LAT instrumental response. This exploits the angular structure imposed by the bimodal structure instead of the radial structure often used. We propose to do this by generating one-dimensional angular power spectra, $\mathcal{P}_{m}$, about each VHEGR source, following the application of an energy-dependent mask to remove the contribution of the direct emission from the central source. These have the considerable advantage that, unlike the images, they retain evidence of angular structure in the image when stacked regardless of absolute orientation.

The presence of bimodal structures in the underlying gamma-ray count maps generally produces a quadrupolar power excess (i.e., at $m=2$ ), with the angular power spectrum oscillating thereafter. The magnitude of the low-power excess and the depth of the oscillations depend on the intrinsic source parameters. Nevertheless, the sawtooth nature is generally present, both among the different IGMF models and intrinsic 
parameter distributions as well as in individual realizations of the images. Importantly, this is qualitatively distinct from the smooth power excesses induced by many potential observational and astronomical contaminants, including undetected neighbors, an unresolved point-source background, large background gradients, failures to center sources properly, and structure in the LAT instrument response.

Via Monte Carlo simulation of the images from VHEGR Fermi sources, we produced an optimized experiment for detecting ICC halos. That is, the ability to generate a large ensemble of realizations of images for the full sample enables the theoretical prediction of the anticipated distribution of the power at each $m$. We therefore optimized both the procedure and sample of Fermi AGNs. Although the procedure is independent of the details underlying the ICC halo generation, the optimal list of Fermi sources is not; we report the optimized source samples for detecting ICC halos associated with largescale, uniform IGMFs in Table 1. It is important to note that apart from the source SEDs and measured redshifts, this was performed entirely using simulated images and thus is not colored by actual structures within the gamma-ray images.

Based upon this optimized experiment, we are able to define confidence levels at which a given halo model can be detected or excluded using current and future Fermi data. The ICC halos generated in the presence of a large-scale, uniform IGMF are detectable at high confidence for IGMF strengths between $10^{-17} \mathrm{G}$ and $10^{-14} \mathrm{G}$, well matched to the range implied by gamma-ray observations of bright, nearby Fermi AGNs. At weaker IGMF strengths, the putative halos are sufficiently spatially concentrated that they are confused with the central source. At stronger IGMF strengths, they are sufficiently spatially diffused that they are confused with neighboring sources. Were Fermi to continue operations for roughly eight more years, the significance with which ICC halos could be detected or excluded would increase.

In a forthcoming companion publication, we apply this to the existing sample of suitable Fermi blazars, placing constraints on the geometry and strength of the IGMF.

A.E.B. and M.S. receive financial support from the Perimeter Institute for Theoretical Physics and the Natural Sciences and Engineering Research Council of Canada through a Discovery Grant. Research at the Perimeter Institute is supported by the Government of Canada through Industry Canada and by the Province of Ontario through the Ministry of Research and Innovation. C.P. acknowledges support by the European Research Council under ERC-CoG grant CRAGSMAN-646955 and by the Klaus Tschira Foundation. P. C. gratefully acknowledges support from the NASA ATP program through NASA grant NNX13AH43G, and NSF grant AST-1255469. A.L. receives financial support from an Alfred P. Sloan Research Fellowship, NASA ATP Grant NNX14AH35G, and NSF Collaborative Research Grant 411920 and CAREER grant 1455342. E.P. acknowledges support by the Kavli Foundation.

\section{Appendix A}

\section{Generalized $\lambda$ Distribution Fit of Intrinsic Opening Angles}

The generalized $\lambda$ distribution $(\mathrm{G} \lambda \mathrm{D})$ provides a convenient parameterization of probability distributions (Ramberg \& Schmeiser 1974). A key feature of the $G \lambda D$ is that it parameterizes the inverse of the cumulative probability distribution instead of the probability density directly. This is particularly useful for generating random variates from the parameterized probability distribution.

The $\mathrm{G} \lambda \mathrm{D}$, with the Ramberg and Schmeiser (RS) parameterization, is defined by four constants, $\lambda_{1,2,3,4}$, in terms of which

$$
F^{-1}(u)=\lambda_{1}+\frac{u^{\lambda_{3}}-(1-u)^{\lambda_{4}}}{\lambda_{2}}
$$

where $u \in[0,1]$ is the cumulative probability and $F^{-1}(u)$ is a map to the dependent variable. Drawing a random variate from the desired distribution is then reduced to choosing a uniform variate on $[0,1]$ and evaluating $F^{-1}(u)$.

We performed a nonparametric fit of the cumulative distribution of intrinsic radio opening angles, $\alpha_{\text {int }}$, from Pushkarev et al. (2009) using the numerical statistics environment R (R Core Team 2014) and the G $\lambda$ D RS fitting package by GLDEX (Su 2016, 2007) and performed a maximum-likelihood fit. A KS test was performed to analyze the quality of the fit, and the result was a $p$ value of 0.9704 , i.e., consistent with the assumption that they are drawn from the same distribution. The four $\mathrm{G} \lambda \mathrm{D}$ parameters are $\lambda_{1}=0.8261001, \quad \lambda_{2}=-0.63510851, \quad \lambda_{3}=$ -0.07892053 , and $\lambda_{4}=-0.2946635$, and the resulting probability distribution was used to generate realizations of the jet population in Section 3.3.

\section{Appendix B Maximum-likelihood Center Finding}

Here, we describe how we produce refined high-accuracy estimates of the source location. We begin with a parameterized model for the probability distribution of photons in an image that describes a Gaussian source on a uniform background. This has three parameters: the source position, $\boldsymbol{\mu}$, the ratio of photons in the background to those in the source, $A$, and the width of the source distribution, $\sigma$. We will assume that the last is fixed by the characteristic size of the Fermi PSF. Finally, and critically, we will only perform the center-fitting within a known circular window with angular radius $R$ about some fixed initial position. Thus, the probability of a given photon being located at a position $\boldsymbol{x}$ given the parameter $\boldsymbol{p}=(\boldsymbol{\mu}, A)$ is

$$
P(\boldsymbol{x} \mid \boldsymbol{p})=\Theta(R-|\boldsymbol{x}|) N\left[\frac{1}{2 \pi \sigma^{2}} e^{-(\boldsymbol{x}-\boldsymbol{\mu})^{2} / 2 \sigma^{2}}+\frac{A}{\pi R^{2}}\right],
$$

These are not all independent, related by the normalization condition upon $P(\boldsymbol{x} \mid \boldsymbol{p})$ :

$$
\begin{aligned}
1= & \int d^{2} x P(\boldsymbol{x} \mid \boldsymbol{p})=\int_{0}^{R} r d r \int_{0}^{2 \pi} d \theta N \\
& \times\left[\frac{1}{2 \pi \sigma^{2}} e^{-\left(r^{2}-2 r \mu \cos \theta+\mu^{2}\right) / 2 \sigma^{2}}+\frac{A}{\pi R^{2}}\right] \\
= & N A+N e^{-\mu^{2} / 2 \sigma^{2}} \int_{0}^{R / \sigma} d x x e^{-x^{2} / 2} I_{0}(x m),
\end{aligned}
$$

where $x \equiv r / \sigma, m \equiv \mu / \sigma$, and $I_{0}(x)$ is the modified Bessel function of the first kind. This final integral is difficult to perform in practice due to the presence of $I_{0}$. It maybe be simplified via various asymptotic expansions, though in practice it is most easily evaluated numerically. 
However it is computed, the resulting integral is a function of $R^{2}, \sigma^{2}$, and $\mu^{2}$ only, and thus we define

$$
I\left(R^{2}, \sigma^{2}, \mu^{2}\right) \equiv e^{-\mu^{2} / 2 \sigma^{2}} \int_{0}^{R / \sigma} d x x e^{-x^{2} / 2} I_{0}(x m) .
$$

Regardless of how $I\left(R^{2}, \sigma^{2}, \mu^{2}\right)$ is computed, the normalization condition becomes

$$
N=\frac{1}{A+I\left(R^{2}, \sigma^{2}, \mu^{2}\right)},
$$

and thus, our probability distribution is

$$
P(\boldsymbol{x} \mid \boldsymbol{p})=\Theta(R-|\boldsymbol{x}|) \frac{\left(\pi R^{2} / 2 \pi \sigma^{2}\right) e^{-(\boldsymbol{x}-\boldsymbol{\mu})^{2} / 2 \sigma^{2}}+A}{\pi R^{2}\left[A+I\left(R^{2}, \sigma^{2}, \mu^{2}\right)\right]} .
$$

The corresponding likelihood for a set of $\left\{\boldsymbol{x}_{j}\right\}$ is then

$$
\begin{aligned}
L\left(\left\{\boldsymbol{x}_{j}\right\} \mid \boldsymbol{p}\right) & =-2 \sum_{j} \log \left\{\frac{\left(\pi R^{2} / 2 \pi \sigma^{2}\right) e^{-\left(\boldsymbol{x}_{j}-\boldsymbol{\mu}\right)^{2} / 2 \sigma^{2}}+A}{\pi R^{2}\left[A+I\left(R^{2}, \sigma^{2}, \mu^{2}\right)\right]}\right\} \\
& =-2 \sum_{j} \log \left(\frac{\Delta_{j}+A}{I+A}\right)+\text { const } .
\end{aligned}
$$

where we ignore the constant $\pi R^{2}$ term and define

$$
\Delta_{j}=\frac{\pi R^{2}}{2 \pi \sigma^{2}} e^{-\left(\boldsymbol{x}_{j}-\boldsymbol{\mu}\right)^{2} / 2 \sigma^{2}}
$$

Finally, to avoid issues with $A$ dropping below zero, we replace it with $e^{\alpha}$, which is positive definite. Thus,

$$
L=-2 \sum_{j} \log \left(\frac{\Delta_{j}+e^{\alpha}}{I+e^{\alpha}}\right)+\text { const. }
$$

We supplement these with the derivatives

$$
\begin{aligned}
\frac{\partial L}{\partial \mu} & =-2 \sum_{j}\left[\frac{\Delta_{j}}{\Delta_{j}+e^{\alpha}} \frac{\left(\boldsymbol{x}_{j}-\boldsymbol{\mu}\right)}{\sigma^{2}}-\frac{2 \boldsymbol{\mu}}{I+e^{\alpha}} \frac{\partial I}{\partial \mu^{2}}\right] \\
\text { and } \frac{\partial L}{\partial \alpha} & =-2 \sum_{j} e^{\alpha}\left[\frac{1}{\Delta_{j}+e^{\alpha}}-\frac{1}{I+e^{\alpha}}\right] .
\end{aligned}
$$

The values of $\boldsymbol{\mu}$ and $\alpha$ are then obtained by numerically minimizing $L$.

\section{Appendix C \\ Example Angular Power Spectra}

Here, we present a number of angular power spectra for toy models. These are primarily for illustrative and contextual purposes-power spectra in the text are obtained for physically motivated mock images in various circumstances. In all cases we make use of the following general definitions. The angular power associated with a given image is

$$
\begin{aligned}
\mathcal{P}_{m} & =\frac{1}{N^{2}}\left|\sum_{j} e^{i m \theta_{j}}\right|^{2}=\frac{1}{N^{2}} \sum_{j, k} e^{i m\left(\theta_{j}-\theta_{k}\right)} \\
& =\frac{1}{N}+\frac{1}{N^{2}} \sum_{j \neq k} e^{i m\left(\theta_{j}-\theta_{k}\right)} .
\end{aligned}
$$

Note that, generally, for $m=0$, we have $\mathcal{P}_{0}=N^{-2} \sum_{j, k} 1=1$ independent of the details of the distribution of $\theta_{j}$.

To compute the statistical properties of $\mathcal{P}_{m}$ for $m>0$, we must specify the probability distribution of $\theta_{N, m}$, which we describe via the probability density of finding a photon at $\theta$, $\wp(\theta)$. (The independence of $m=0$ is a consequence of the normalization condition on $\wp(\theta)$.) Hence, assuming each photon is independent, the mean is given by

$$
\begin{aligned}
\left\langle\mathcal{P}_{m}\right\rangle & =\int d \theta_{1} \ldots d \theta_{N} \wp\left(\theta_{1}\right) \ldots \wp\left(\theta_{N}\right)\left[\frac{1}{N}+\frac{1}{N^{2}} \sum_{j \neq k} e^{i m\left(\theta_{j}-\theta_{k}\right)}\right] \\
& =\frac{1}{N}+\frac{N-1}{N} \int d \theta_{1} d \theta_{2} \wp\left(\theta_{1}\right) \wp\left(\theta_{2}\right) e^{i m\left(\theta_{1}-\theta_{2}\right)} \\
& =\frac{1}{N}+\frac{N-1}{N}\left|\varphi_{m}\right|^{2},
\end{aligned}
$$

where $\varphi_{m}$ is the characteristic function of the photon distribution,

$$
\varphi_{m} \equiv \int d \theta \wp(\theta) e^{i m \theta} .
$$

The variance may be computed in a similar way, though some care must be taken with counting the degeneracies of the various terms, ultimately yielding for $m>0$

$$
\begin{aligned}
& \sigma_{\mathcal{P}_{m}}^{2}=\left\langle\mathcal{P}_{m}^{2}\right\rangle-\left\langle\mathcal{P}_{m}\right\rangle^{2}=\frac{1}{N^{4}} \int d \theta_{1} \ldots d \theta_{N} \wp\left(\theta_{1}\right) \ldots \wp\left(\theta_{N}\right) \\
& \times\left[N^{2}+2 N \sum_{j \neq k} e^{i m\left(\theta_{j}-\theta_{k}\right)}+\sum_{j \neq k, a \neq b} e^{i m\left(\theta_{j}-\theta_{k}+\theta_{a}-\theta_{b}\right)}\right] \\
& -\left[N+N(N-1)\left|\phi_{m}\right|^{2}\right]^{2}=\frac{1}{N^{4}} \int d \theta_{1} \ldots d \theta_{N} \wp\left(\theta_{1}\right) \ldots \wp\left(\theta_{N}\right) \\
& \times\left[N^{2}+2 N \sum_{j \neq k} e^{i m\left(\theta_{j}-\theta_{k}\right)}+\sum_{j=b \neq k=a} 1+\sum_{j=b \neq k \neq a} e^{i m\left(\theta_{a}-\theta_{k}\right)}\right. \\
& +\sum_{j \neq b \neq k=a} e^{i m\left(\theta_{j}-\theta_{b}\right)}+\sum_{j=a \neq k=b} e^{i m\left(2 \theta_{j}-2 \theta_{k}\right)}+\sum_{j \neq b} e^{i m\left(2 \theta_{j}-\theta_{k}-\theta_{b}\right)} \\
& \left.+\sum_{j \neq a \neq k=b} e^{i m\left(\theta_{j}-2 \theta_{k}+\theta_{a}\right)}+\sum_{j \neq k \neq a \neq b} e^{i m\left(\theta_{j}-\theta_{k}+\theta_{a}-\theta_{b}\right)}\right] \\
& -\left[N+N(N-1)\left|\phi_{m}\right|^{2}\right]^{2} \\
& =\frac{1}{N^{4}}\left[N^{2}+2 N^{2}(N-1)\left|\varphi_{m}\right|^{2}+N(N-1)\right. \\
& +2 N(N-1)(N-2)\left|\varphi_{m}\right|^{2}+N(N-1)\left|\varphi_{2 m}\right|^{2}+N(N-1)(N-2) \\
& \times\left(\varphi_{2 m}^{*} \varphi_{m}^{2}+\varphi_{2 m} \varphi_{m}^{* 2}\right)+N(N-1)(N-2)(N-3)\left|\varphi_{m}\right|^{4} \\
& \left.-N^{2}-2 N^{2}(N-1)\left|\varphi_{m}\right|^{2}-N^{2}(N-1)^{2}\left|\varphi_{m}\right|^{4}\right] \\
& =\frac{N-1}{N^{3}}\left[1+(2 N-4)\left|\varphi_{m}\right|^{2}-(4 N-6)\left|\varphi_{m}\right|^{4}\right. \\
& \left.+\left|\varphi_{2 m}\right|^{2}+(N-2)\left(\varphi_{2 m}^{*} \varphi_{m}^{2}+\varphi_{2 m} \varphi_{m}^{* 2}\right)\right] .
\end{aligned}
$$

Identifying $\left\langle\mathcal{P}_{m}\right\rangle$ and $\sigma_{\mathcal{P}_{m}}$ for specific models now requires the specification of $\varphi_{m}$. We consider a handful of specific cases below.

\section{C.1. Isotropic Background}

We begin with an isotropic background, i.e.,

$$
\wp(\theta)=\frac{1}{2 \pi} \Rightarrow \varphi_{m}=\delta_{m 0},
$$


and thus for $m>0$,

$$
\left\langle\mathcal{P}_{m}\right\rangle=\frac{1}{N} \quad \text { and } \quad \sigma_{\mathcal{P}_{m}}^{2}=\frac{N-1}{N^{3}}
$$

Note that this does not imply a uniform background, only one that is isotropic about the chosen image center. This is simply the Poisson noise limit, and as is typical in power spectrum estimation, based on a single source, the variance in the estimate of $\mathcal{P}_{m}$ is comparable to its mean.

\section{C.2. Offset Gaussian}

We now consider a peaked source offset from the origin, as may occur if the source location estimate is in error. Here we make the simplifying assumption that the source is Gaussian, i.e., we set the two-dimensional photon probability density to be

$$
\begin{array}{r}
\wp(\alpha, \delta)=\frac{1}{2 \pi \sigma^{2}} e^{-\left[(\alpha-\eta \sigma)^{2}+\delta^{2}\right] / 2 \sigma^{2}}=\frac{1}{2 \pi \sigma^{2}} e^{-\left(r^{2}+\eta^{2} \sigma^{2}\right) / 2 \sigma^{2}} \\
\times\left(1+\frac{\eta r \cos \theta}{\sigma}+\frac{\eta^{2} r^{2} \cos ^{2} \theta}{2 \sigma^{2}}+\frac{\eta^{3} r^{3} \cos ^{3} \theta}{6 \sigma^{3}}+\ldots\right) .
\end{array}
$$

Integrating over radius, this gives the needed angular probability density

$$
\begin{aligned}
\wp(\theta)= & \int r d r \wp(\alpha, \delta)=\frac{1}{2 \pi}\left[1+\sqrt{\frac{\pi}{2}} \eta \cos \theta\right. \\
& \left.+\eta^{2} \cos ^{2} \theta+\sqrt{\frac{\pi}{8}} \eta^{3} \cos ^{3} \theta+\ldots\right] .
\end{aligned}
$$

Therefore, the characteristic function is

$$
\begin{aligned}
\varphi_{m}= & \delta_{m 0}+\frac{1}{2} \sqrt{\frac{\pi}{2}} \eta\left(\delta_{m 1}+\delta_{m-1}\right) \\
& +\frac{1}{4} \eta^{2}\left(\delta_{m 2}+2 \delta_{m 0}+2 \delta_{m-2}\right) \\
& +\frac{1}{8} \sqrt{\frac{\pi}{8}} \eta^{3}\left(\delta_{m 3}+3 \delta_{m 1}+3 \delta_{m-1}+\delta_{m-3}\right)+\ldots
\end{aligned}
$$

To lowest order in $\eta$ at each $m$, this gives

$$
\begin{gathered}
\left\langle\mathcal{P}_{m}\right\rangle=N+N(N-1)\left[\delta_{m 0}+\frac{\pi}{8} \eta^{2} \delta_{m 1}\right. \\
\left.+\frac{1}{16} \eta^{4} \delta_{m 2}+\frac{\pi}{512} \eta^{6} \delta_{m 3}+\ldots\right] .
\end{gathered}
$$

Note that the contribution to the high- $m$ multipoles falls off as $\eta^{2 m}$. Not surprisingly, the variance also falls off quite rapidly in $\eta:$

$$
\begin{aligned}
& \sigma_{\mathcal{P}_{m}}^{2} \simeq \sum_{j} 2 N^{3}\left[\delta_{m 0}+\frac{\pi}{8} \eta^{2} \delta_{m 1}+\frac{1}{16} \eta^{4} \delta_{m 2}\right. \\
& \left.+\frac{\pi}{512} \eta^{6} \delta_{m 3}+\ldots\right] .
\end{aligned}
$$

We can construct a general formula using the integral

$$
\int_{0}^{\infty} d r r^{n+1} e^{-r^{2} / 2}=2^{n / 2} \Gamma\left(\frac{n}{2}+1\right),
$$

with which we have

$$
\begin{aligned}
\wp(\theta) & =\frac{1}{2 \pi} \sum_{j=0}^{\infty} \frac{2^{j / 2}}{j !} \Gamma\left(\frac{j}{2}+1\right) \eta^{j} \cos ^{j} \theta \Rightarrow \\
\varphi_{m} & =\sum_{j=0}^{\infty} \frac{2^{-j / 2}}{j !} \Gamma\left(\frac{j}{2}+1\right) \eta^{j} \sum_{k=-j}^{j} \frac{j !}{k !(j-k) !} \delta_{m k},
\end{aligned}
$$

in terms of which we have the lowest-order terms in $\eta$ for each $m$ in the power spectrum

$$
\begin{gathered}
\left\langle\mathcal{P}_{m}\right\rangle \approx\left|\varphi_{m}\right|^{2} \approx 2^{-m}\left[\frac{1}{m !} \Gamma\left(\frac{m}{2}+1\right)\right]^{2} \eta^{2 m}<2^{-3 m / 2} \eta^{2 m} \\
\times \text { and } \sigma_{\mathcal{P}_{m}}^{2} \approx 2\left|\phi_{m}\right|^{2}<2^{1-3 m / 2} \eta^{2 m}
\end{gathered}
$$

\section{C.3. Weak Image Components}

There are many instances where the image is comprised of a dominant, isotropic component and a subdominant structured component. In this case, we generally have

$$
\wp(\theta)=\frac{1-\eta}{2 \pi}+\eta h(\theta) \Rightarrow \varphi_{m}=\delta_{m 0}+\eta \psi_{m},
$$

where $\psi_{m}$ is the structure function associated with the unitnormalized $h(\theta)$ perturbing distribution, with the strength described by the order parameter $\eta \ll 1$ that is equal to the ratio of the photons associated with the perturbation to those in the isotropic background. Then, for $m>0$,

$$
\left\langle\mathcal{P}_{m}\right\rangle \approx \frac{1}{N}+\eta^{2}\left|\psi_{m}\right|^{2} \quad \text { and } \quad \sigma_{\mathcal{P}_{m}}^{2} \approx \frac{1}{N^{2}}+\frac{2}{N} \eta^{2}\left|\psi_{m}\right|^{2} .
$$

Note that again $\sigma_{\mathcal{P}_{m}} \approx \mathcal{P}_{m}$, which motivates combining multiple estimates of $\mathcal{P}_{m}$ to obtain an improved statistical significance of any detection.

\section{C.3.1. Gaussian Neighbor}

Distant peaked sources, arising from unidentified neighbors, will also induce dipolar power. Again, we make the simplifying assumption that the neighboring source is Gaussian, which, if it is sufficiently far, is approximately described by

$$
h(\theta)=\frac{e^{-\left(\theta-\theta_{0}\right)^{2} / 2 w^{2}}}{\sqrt{2 \pi} w} \Rightarrow \psi_{m}=e^{i m \theta_{0}-w^{2} m^{2} / 2},
$$

where $\theta_{0}$ and $w=\sigma / \Delta r$ are the location and width of the companion in polar angle measured about the central source. The associated mean power spectrum is

$$
\left\langle\mathcal{P}_{m}\right\rangle \approx \frac{1}{N}+\eta^{2} e^{-w^{2} m^{2}} \quad \text { and } \quad \sigma_{\mathcal{P}_{m}}^{2} \approx \frac{1}{N^{2}}+\frac{2}{N} \eta^{2} e^{-w^{2} m^{2}} .
$$

As anticipated, this generates substantial dipolar power, smoothly falling off on a scale inversely set by the width.

\section{C.3.2. Bimodal Gaussian}

As an approximation to what the angular power spectrum from a bimodal halo may look like, we consider a bimodal 
Gaussian consisting of two sources located $180^{\circ}$ apart. That is,

$$
\begin{aligned}
h(\theta) & =\frac{1}{\sqrt{8 \pi} w}\left(e^{-\left(\theta-\theta_{0}\right)^{2} / 2 w^{2}}+e^{-\left(\theta-\theta_{0}+\pi\right)^{2} / 2 w^{2}}\right) \\
\Rightarrow \psi_{m} & =\Delta_{m} e^{i m \theta_{0}-w^{2} m^{2} / 2},
\end{aligned}
$$

where $\Delta_{m}$ is unity for even $m$ and zero for odd $m$. This is similar to the Gaussian neighbor, with the exception of the factor of $\Delta_{m}$ that arises from the beating between the two sources. Hence, the mean power spectrum is

$$
\begin{aligned}
\left\langle\mathcal{P}_{m}\right\rangle & \approx \frac{1}{N}+\eta^{2} \Delta_{m} e^{-w^{2} m^{2}} \\
\text { and } \quad \sigma_{\mathcal{P}_{m}}^{2} & \approx \frac{1}{N^{2}}+\frac{2}{N} \eta^{2} \Delta_{m} e^{-w^{2} m^{2}} .
\end{aligned}
$$

Note that this exhibits the clear sawtooth indicative generally of the bimodal structures.

\section{Appendix D \\ Estimate of PSF-induced Angular Structure}

We make a quantitative estimate of the impact of the intrinsic anisotropy of the Fermi LAT PSF. To do this, we follow the description in Section 5.2.3 of Ackermann et al. (2012a) and adopt an azimuthal dependence for the PSF of the form

$$
f(\theta)=1+q_{0} \xi^{q_{1}} \text { where } \xi=\frac{4}{\pi}\left|\left(\theta \bmod \frac{\pi}{2}\right)-\frac{\pi}{4}\right|,
$$

where $q_{0}, q_{1}$, and $\xi$ have the same meaning as in Equation (15) of Ackermann et al. (2012a). The impact of this is to modify the distribution of photons in the image, inducing a small $m=4$ perturbation on the angular probability distribution.

The gamma rays from a bright Fermi AGN in a typical image are accumulated over many years, and therefore the LAT presents different orientations. Were the orientation completely independent for each photon, the net PSF would become isotropic and therefore any remaining structure would be absent. However, we may imagine that subsequent photons have LAT orientations that are closely correlated. The roll angle of the LAT changes primarily as a result of the fixed telescope orientation relative to the Sun. The square geometry of the LAT implies that the angular impact on the PSF is effectively averaged by the time it has rotated by $45^{\circ}$, corresponding to roughly 45 days. Over this timescale, a non-varying Fermi source would accumulate less than $2 \%$ of its total fluence or roughly 100 photons.

Thus, we estimate the residual power from $n \approx 50$ epochs of $N \approx 100$ events, assuming perfect correlation in the LAT orientation for the latter. Within each subgroup there is a fixed roll angle, $\vartheta_{\mu}$, and hence relative to the orientation of the LAT the polar angles of the photons are $\theta_{j, \mu}=\theta_{j}-\vartheta_{\mu}$. Between subgroups, the roll angle is effectively uncorrelated. Thus, for $m>0$,

$$
\begin{aligned}
\left\langle\mathcal{P}_{m}\right\rangle & =\frac{1}{(n N)^{2}} \sum_{\mu, \nu} \sum_{j, k}\left\langle e^{i m\left(\theta_{j}-\theta_{k}\right)} e^{-i m\left(\vartheta_{j}+\vartheta_{k}\right)}\right\rangle \\
& =\frac{1}{n N^{2}} \sum_{j, k}\left\langle e^{i m\left(\theta_{j}-\theta_{k}\right)}\right\rangle,
\end{aligned}
$$

where the independence of different $\vartheta_{\mu}$ was employed.
The prefactor of $n^{-1}$ is simply the angular power spectrum for the single epoch, for which the photon distribution is perturbed from isotropy. This is precisely the case considered in Appendix C.3. Here, the perturbation is $h(\theta)=\xi / \pi$, where we have set $q_{1}=1$ for simplicity and implicitly subsumed $q_{0}$ into $\eta=2 q_{0}$. The associated characteristic function is

$$
\begin{aligned}
\psi_{m}= & \int d \theta \frac{\xi}{\pi} e^{i m \theta}=\left(1+e^{i m \pi / 2}+e^{i m \pi}+e^{3 i m \pi / 2}\right) \\
& \times \int_{0}^{\pi / 2} d \theta \frac{\xi}{\pi} e^{i m \theta}=-\frac{i}{2} \Sigma_{m} e^{i m \pi / 4} j_{1}\left(\frac{m \pi}{4}\right),
\end{aligned}
$$

where $\Sigma_{m}$ is unity for $m$ that are divisible by 4 and zero otherwise, and $j_{1}(x)$ is the spherical Bessel function of the first kind of order 1 . Therefore, the corresponding mean angular power spectrum is

$$
\left\langle\mathcal{P}_{m}\right\rangle=\frac{1}{n}\left[\frac{1}{N}+\eta^{2} \Sigma_{m} \frac{1}{4} j_{1}^{2}\left(\frac{m \pi}{4}\right)\right] \lesssim \frac{1}{n N}+\frac{\eta^{2}}{n} \frac{\Sigma_{m}}{4 \pi^{2} m^{2}} .
$$

The excess is non-vanishing only for $m=4$ and its harmonics; in particular, there is no power at $m=2$. For a typical value of $\eta \approx 0.2$, corresponding to $q_{0} \approx 0.1$, the excess at $m=4$ is $\lesssim 10^{-6}$, which is much smaller than $(n N)^{-1} \gtrsim 10^{-4}$.

In practice, the angular power associated with structure in the PSF is further reduced by the fraction of total photons due to the source. This is impacted by both the surface brightness of the background, typically contributing $25 \%$ for the brightest sources, and the source mask, typically cutting the source contribution by half. This makes only an order of unity correction.

\section{Appendix E Source Sample}

Here we collect the images and fits for the 18 sources listed in Table 1 that comprise the full set of sources within the optimized source lists. Details of the fitting process are described in Section 3.1; we only summarize these here.

There were 84 sources selected on the basis of their $2 \mathrm{FHL}$ $50 \mathrm{GeV}-2 \mathrm{TeV}$ flux, as well as having a redshift below 0.5 and a 3LAC counterpart. Gamma-ray SEDs were produced by compiling the $0.1-0.3 \mathrm{GeV}, 0.3-1 \mathrm{GeV}, 1-3 \mathrm{GeV}, 3-10 \mathrm{GeV}$, and $10-100 \mathrm{GeV}$ flux measurements reported in $3 \mathrm{LAC}$ and 50-171 GeV, $171-585 \mathrm{GeV}$, and $585-2000 \mathrm{GeV}$ reported in 2FHL. These were deabsorbed via Equation (8), which was evaluated at the geometric center of the energy bin. A maximum-likelihood fit of the broken power-law SED model was performed for each candidate source, described by lowand high-energy spectral indexes $\Gamma_{l}$ and $\Gamma_{h}$, a pivot energy $E_{p}$, and a normalization.

Sources for which the source-frame $\mathrm{TeV}$ flux, i.e., $E^{2} d N / d E$ at $(1+z) \mathrm{TeV}$, was above $10^{-9} \mathrm{GeV} \mathrm{cm}^{-2} \mathrm{~s}^{-1}$ were then visually inspected for neighboring sources within $2^{\circ}$ or large background gradients. Sufficiently isolated $\mathrm{TeV}$-bright sources comprise the source sample employed here, consisting of the 27 objects listed in Table 2 . Note that the sources that comprise the optimized source lists are chosen from these 27 after simulating the ability to detect ICC halos (see Section 4.6).

The observed and deabsorbed SEDs and our fits for the 18 sources used here are summarized in Figure 20 and explicitly shown in Figures 21 and 22. 

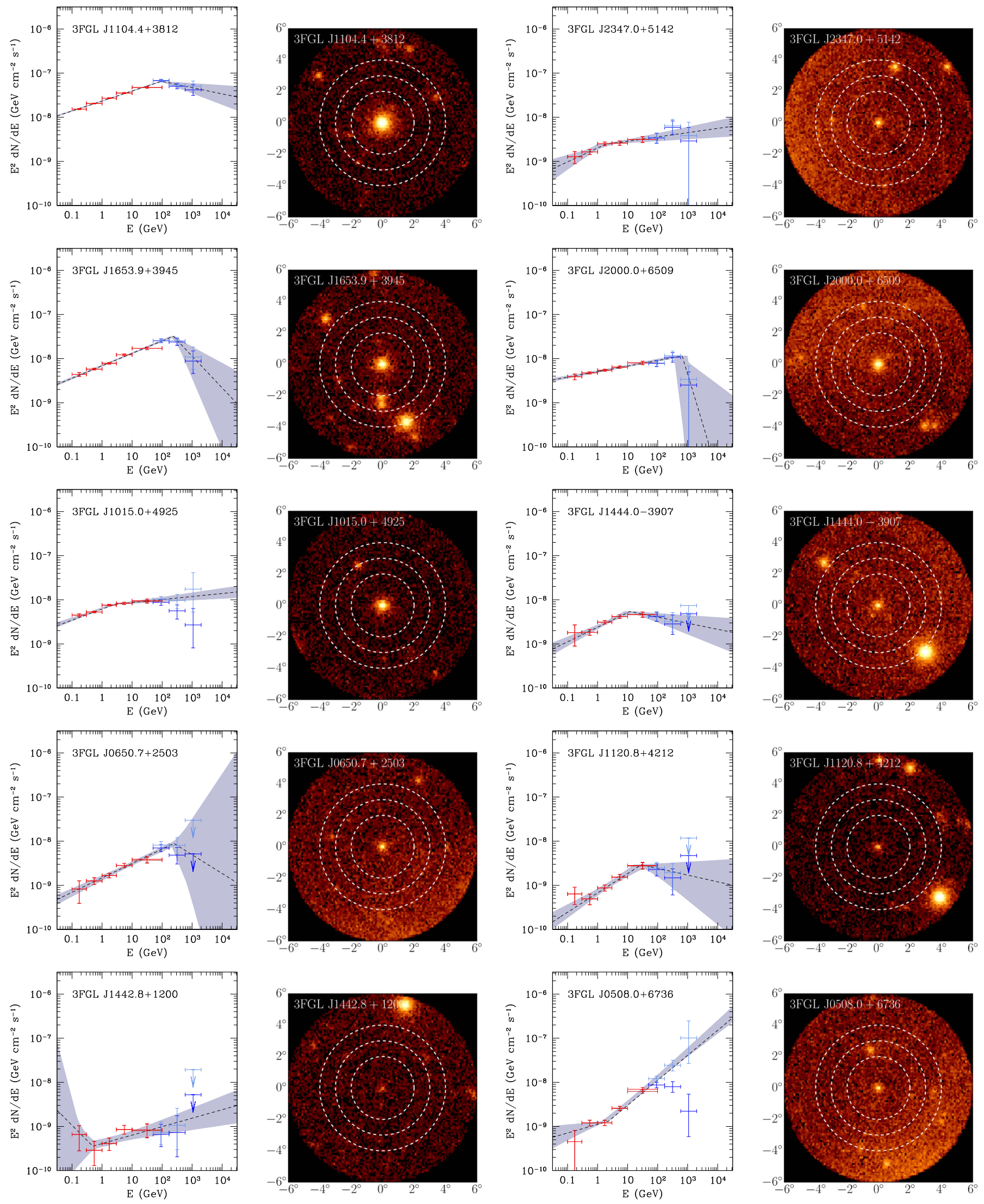

Figure 21. Source images and SEDs for objects in Table 1-part 1. Left: compilations of the 3FGL (red/orange) and 2FHL (blue/light blue) fluxes. Also shown are the broken power-law fits (dashed lines) and associated uncertainty (gray region). Right: 1-100 GeV count maps for the sources on the left. 

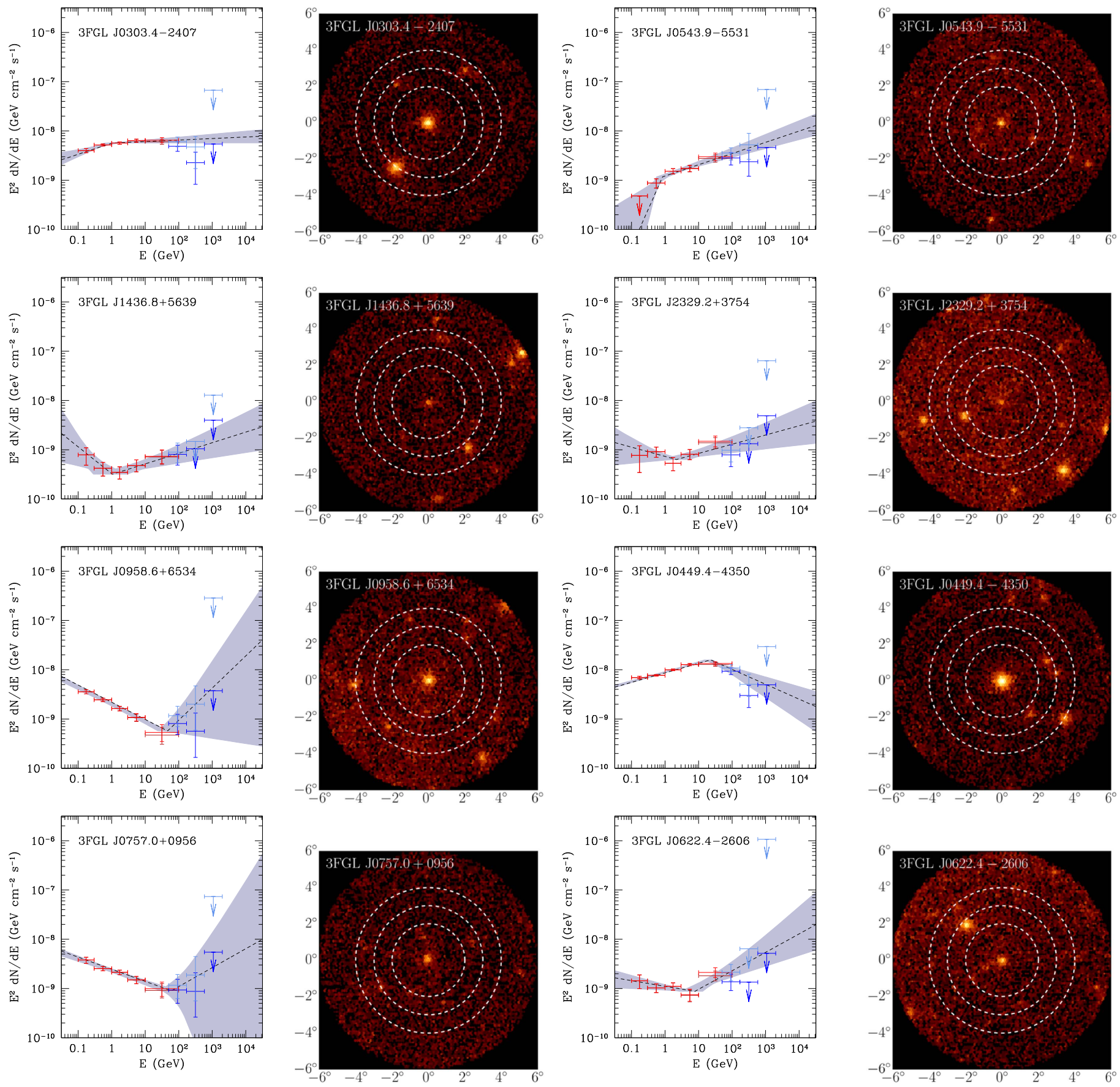

Figure 22. Source images and SEDs for objects in Table 1—part 2. Left: compilations of the 3FGL (red/orange) and 2FHL (blue/light blue) fluxes. Also shown are the broken power-law fits (dashed lines) and associated uncertainty (gray region). Right: 1-100 GeV count maps for the sources on the left.

\section{ORCID iDs}

Avery E. Broderick iㅣ https://orcid.org/0000-0002-3351-760X Mohamad Shalaby (i) https://orcid.org/0000-0001-9625-5929 Christoph Pfrommer (1) https://orcid.org/0000-00027275-3998

Philip Chang $\mathbb{1}$ https://orcid.org/0000-0002-2137-2837

Astrid Lamberts (i) https://orcid.org/0000-0001-8740-0127

\section{References}

Abdo, A. A., Ackermann, M., Ajello, M., et al. 2010a, ApJS, 188, 405 Abdo, A. A., Ackermann, M., Ajello, M., et al. 2010b, PhRvL, 104, 101101
Abdo, A. A., Ackermann, M., Agudo, I., et al. 2010c, ApJ, 716, 30 Acero, F., Ackermann, M., Ajello, M., et al. 2015, ApJS, 218, 23 Ackermann, M., Ajello, M., Albert, A., et al. 2012a, ApJS, 203, 4 Ackermann, M., Ajello, M., Albert, A., et al. 2015a, ApJ, 799, 86 Ackermann, M., Ajello, M., Albert, A., et al. 2016b, PhRvL, 116, 151105 Ackermann, M., Ajello, M., Allafort, A., et al. 2011, ApJ, 743, 171 Ackermann, M., Ajello, M., Allafort, A., et al. 2012b, sci, 338, 1190 Ackermann, M., Ajello, M., Allafort, A., et al. 2013a, ApJ, 765, 54 Ackermann, M., Ajello, M., Allafort, A., et al. 2013b, ApJS, 209, 34 Ackermann, M., Ajello, M., Atwood, W. B., et al. 2015b, ApJ, 810, 14 Ackermann, M., Ajello, M., Atwood, W. B., et al. 2016a, ApJS, 222, 5 Albert, J., Aliu, E., Anderhub, H., et al. 2007, ApJ, 663, 125

Alexander, P., \& Leahy, J. P. 1987, MNRAS, 225, 1

Ando, S., \& Kusenko, A. 2010, ApJL, 722, L39

Böttcher, M., Reimer, A., Sweeney, K., \& Prakash, A. 2013, ApJ, 768, 54 
Broderick, A. E., Chang, P., \& Pfrommer, C. 2012, ApJ, 752, 22

Broderick, A. E., Pfrommer, C., Puchwein, E., \& Chang, P. 2014a, ApJ, 790, 137

Broderick, A. E., Pfrommer, C., Puchwein, E., Chang, P., \& Smith, K. M. 2014b, ApJ, 796, 12

Broderick, A. E., Tiede, P., Shalaby, M., et al. 2016, ApJ, 832, 109

Cerruti, M., Zech, A., Boisson, C., \& Inoue, S. 2015, MNRAS, 448, 910

Chang, P., Broderick, A. E., \& Pfrommer, C. 2012, ApJ, 752, 23

Chang, P., Broderick, A. E., Pfrommer, C., et al. 2014, ApJ, 797, 110

Chang, P., Broderick, A. E., Pfrommer, C., et al. 2016, ApJ, 833, 118

Chen, W., Buckley, J. H., \& Ferrer, F. 2015, PhRvL, 115, 211103

Dermer, C. D., Cavadini, M., Razzaque, S., et al. 2011, ApJL, 733, L21

Dolag, K., Kachelriess, M., Ostapchenko, S., \& Tomàs, R. 2011, ApJL, 727, L4

Domínguez, A., Finke, J. D., Prada, F., et al. 2013, ApJ, 770, 77

Duplessis, F., \& Vachaspati, T. 2017, JCAP, 05, 005

Durrer, R., \& Neronov, A. 2013, A\&ARv, 21, 62

Fossati, G., Maraschi, L., Celotti, A., Comastri, A., \& Ghisellini, G. 1998, MNRAS, 299, 433

Ghisellini, G., Celotti, A., Fossati, G., Maraschi, L., \& Comastri, A. 1998, MNRAS, 301, 451

Gould, R. J., \& Schréder, G. P. 1967, PhRv, 155, 1408

H.E.S.S. Collaboration, Abramowski, A., Aharonian, F., et al. 2014, A\&A, 562, A145

Hopkins, P. F., Richards, G. T., \& Hernquist, L. 2007, ApJ, 654, 731

Ichiki, K., Takahashi, K., Ohno, H., Hanayama, H., \& Sugiyama, N. 2006, Sci, 311,827

Kandus, A., Kunze, K. E., \& Tsagas, C. G. 2011, PhR, 505, 1

Lamberts, A., Chang, P., Pfrommer, C., et al. 2015, ApJ, 811, 19

Long, A. J., \& Vachaspati, T. 2015, JCAP, 9, 065

McNamara, B. R., Nulsen, P. E. J., Wise, M. W., et al. 2005, Natur, 433, 45

Miniati, F., \& Elyiv, A. 2013, ApJ, 770, 54

Neronov, A., Semikoz, D., Kachelriess, M., Ostapchenko, S., \& Elyiv, A. 2010, ApJL, 719, L130

Neronov, A., \& Semikoz, D. V. 2009, PhRvD, 80, 123012

Neronov, A., Semikoz, D. V., Tinyakov, P. G., \& Tkachev, I. I. 2011, A\&A, 526,90
Neronov, A., \& Vovk, I. 2010, Sci, 328, 73

Nulsen, P. E. J., McNamara, B. R., Wise, M. W., \& David, L. P. 2005, ApJ, 628,629

Pfrommer, C., Chang, P., \& Broderick, A. E. 2012, ApJ, 752, 24

Prokhorov, D. A., \& Moraghan, A. 2016, MNRAS, 457, 2433

Puchwein, E., Pfrommer, C., Springel, V., Broderick, A. E., \& Chang, P. 2012, MNRAS, 423, 149

Pushkarev, A. B., Kovalev, Y. Y., Lister, M. L., \& Savolainen, T. 2009, A\&A, 507, L33

R Development Core Team 2014 R: A Language and Environment for Statistical Computing (Vienna: R Foundation for Statistical Computing)

Ramberg, J. S., \& Schmeiser, B. W. 1974, Commun. ACM, 17, 78

Ryu, D., Schleicher, D. R. G., Treumann, R. A., Tsagas, C. G., \& Widrow, L. M. 2012, SSRv, 166, 1

Salamon, M. H., \& Stecker, F. W. 1998, ApJ, 493, 547

Schlickeiser, R., Ibscher, D., \& Supsar, M. 2012, ApJ, 758, 102

Schlickeiser, R., Krakau, S., \& Supsar, M. 2013, ApJ, 777, 49

Shabala, S. S., Ash, S., Alexander, P., \& Riley, J. M. 2008, MNRAS, 388, 625

Shalaby, M., Broderick, A. E., Chang, P., et al. 2017, ApJ, 848, 81

Sironi, L., \& Giannios, D. 2014, ApJ, 787, 49

Su, S. 2007, Journal of Statistical Software, 21, 9

Su, S. 2016, GLDEX: Fitting Single and Mixture of Generalised Lambda Distributions (RS and FMKL) using Various Methods, https://rdrr.io/ cran/GLDEX/

Takahashi, K., Mori, M., Ichiki, K., \& Inoue, S. 2012, ApJL, 744, L7

Tavecchio, F., Ghisellini, G., Bonnoli, G., \& Foschini, L. 2011, MNRAS, 414, 3566

Tavecchio, F., Ghisellini, G., Foschini, L., et al. 2010, MNRAS, 406, L70

Taylor, A. M., Vovk, I., \& Neronov, A. 2011, A\&A, 529, A144

Tiede, P., Broderick, A. E., Shalaby, M., et al. 2017, arXiv:1702.02586

Tsagas, C. G., \& Kandus, A. 2005, PhRvD, 71, 123506

Turner, M. S., \& Widrow, L. M. 1988, PhRvD, 37, 2743

Urry, C. M., \& Padovani, P. 1995, PASP, 107, 803

Wakely, S. P., \& Horan, D. 2008, Proc. ICRC (Merida), 3, 1341

Widrow, L. M., Ryu, D., Schleicher, D. R. G., et al. 2012, SSRv, 166, 37

Zacharias, M., \& Wagner, S. J. 2016, A\&A, 588, A110 